\title{
Moduli Spaces of Curves and Representation Theory
}

\author{
E. Arbarello ${ }^{1}$, C. De Concini ${ }^{2}$, V. G. Kac ${ }^{3}$, and C. Procesi ${ }^{1}$ \\ 1 Dipartimento di Matematica, Universita Degli Studi di Roma "La Sapienza", Rome, Italy \\ 2 Dipartimento di Matematica, Universita di Roma II "Tor Vergata", Rome, Italy \\ 3 Department of Mathematics, M.I.T., Cambridge, MA 02139, USA
}

\begin{abstract}
We establish a canonical isomorphism between the second cohomology of the Lie algebra of regular differential operators on $\mathbb{C}^{\times}$of degree $\leqq 1$, and the second singular cohomology of the moduli space $\widehat{\mathscr{F}}_{g-1}$ of quintuples $(C, p, z, L,[\varphi])$, where $C$ is a smooth genus $g$ Riemann surface, $p$ a point on $C, z$ a local parameter at $p, L$ a degree $g-1$ line bundle on $C$, and $[\varphi]$ a class of local trivializations of $L$ at $p$ which differ by a non-zero factor. The construction uses an interplay between various infinite-dimensional manifolds based on the topological space $H$ of germs of holomorphic functions in a neighborhood of 0 in $\mathbb{C}^{\times}$and related topological spaces. The basic tool is a canonical map from $\widehat{\mathscr{F}}_{g-1}$ to the infinite-dimensional Grassmannian of subspaces of $H$, which is the orbit of the subspace $H_{-}$of holomorphic functions on $\mathbb{C}^{\times}$vanishing at $\infty$, under the group Aut $H$. As an application, we give a Lie-algebraic proof of the Mumford formula: $\lambda_{n}=\left(6 n^{2}-6 n+1\right) \lambda_{1}$, where $\lambda_{n}$ is the determinant line bundle of the vector bundle on the moduli space of curves of genus $g$, whose fiber over $C$ is the space of differentials of degree $n$ on $C$.
\end{abstract}

\section{Introduction}

Consider the Lie algebra $\mathscr{D}^{F}$ ( $F$ for finite) of regular differential operators of degree less than or equal to 1 on $\mathbb{C}^{\times}$and its subalgebra $\mathbf{d}^{F}$ of vector fields, so that $\left\{z^{j}, d_{j}=z^{j+1} \frac{d}{d z}\right\}_{j \in \mathbb{Z}}$ is a basis of $\mathscr{D}^{F}$ and $\left\{d_{j}\right\}_{n \in \mathbb{Z}}$ is a basis of $\mathbf{d}^{F}$. The Lie algebra $\mathscr{D}^{F}$ acts in a natural way on the space $V_{n}$ of regular differentials of degree $n$ on $\mathbb{C}^{\times}$with basis $v_{k}=z^{-k} d z^{n}, k \in \mathbb{Z}$. This gives an inclusion

$$
\phi_{n}: \mathscr{D}^{F} \rightarrow \mathbf{a}_{\infty}^{F},
$$

where $\mathbf{a}_{\infty}^{F}$ is the Lie algebra of matrices $\left(a_{i j}\right)_{i, j \in \mathbb{Z}}$ such that $a_{i j}=0$ for $|i-j| \gg 0$. We also consider the restriction of $\phi_{n}$ to $\mathbf{d}^{F}$ :

$$
\varrho_{n}: \mathbf{d}^{F} \rightarrow \mathbf{a}_{\infty}^{F} .
$$


One has the following 2-cocycle on $\mathbf{a}_{\infty}^{F}[\mathrm{KP}, \mathrm{DJKM}]$ :

$$
\begin{cases}\psi\left(E_{i j}, E_{j i}\right)=-\psi\left(E_{j i}, E_{i j}\right)=1 & \text { if } i \leqq 0, \quad j>0 \\ \psi\left(E_{i j}, E_{r s}\right)=0 & \text { otherwise, }\end{cases}
$$

whose cohomology class generates $H_{\text {cont }}^{2}\left(\mathbf{a}_{\infty}^{F}, \mathbb{C}\right) \cong \mathbb{C}$.

Another way of expressing this cocycle is the following. Given $\left(a_{i j}\right) \in \mathbf{a}_{\infty}^{F}$, write $f(z, w)=\sum a_{i j} z^{i-1} w^{-j}$ and let

$$
f_{-+}=\sum_{\substack{i \leqq 0 \\ j>0}} a_{i j} z^{i-1} w^{-j}, \quad f_{+-}=\sum_{\substack{i>0 \\ j \leqq 0}} a_{i j} z^{i-1} w^{-j} .
$$

Both $f_{+-}$and $f_{-+}$are polynomials, and given

$$
g=\sum b_{i j} z^{i-1} w^{-j}, \quad\left(b_{i j}\right) \in \mathbf{a}_{\infty}^{F},
$$

we have

$$
\psi\left(\left(a_{i j}\right),\left(b_{i j}\right)\right)=\operatorname{Res}_{\substack{z=0 \\ w=0}}\left(f_{-+} g_{+-}-g_{-+} f_{+-}\right) .
$$

Pulling back the cocycle $\psi$ via $\phi_{n}$ we get a cocycle $\phi_{n}^{*}(\psi)$ on $\mathscr{D}^{F}$ which works out to be

$$
\begin{aligned}
& \phi_{n}^{*}(\psi)\left(d_{j}, d_{k}\right)=-\delta_{j,-k} \frac{j^{3}-j}{6}\left(6 n^{2}-6 n+1\right), \\
& \phi_{n}^{*}(\psi)\left(z^{j}, z^{k}\right)=\delta_{j,-k} j, \\
& \phi_{n}^{*}(\psi)\left(z^{j}, d_{k}\right)=-\delta_{j,-k}\left(n-\frac{1}{2}\right) j(j-1) .
\end{aligned}
$$

Restricting to $\mathbf{d}^{F}$ we get cocycles $\varrho_{n}^{*}(\psi)$ which satisfy the relation

$$
\varrho_{n}^{*}(\psi)=\left(6 n^{2}-6 n+1\right) \varrho_{0}^{*}(\psi) .
$$

Recall that the cohomology class of $\varrho_{0}^{*}(\psi)$ generates $H^{2}\left(\mathbf{d}^{F}, \mathbb{C}\right) \cong \mathbb{C}$; a less wellknown fact is that $H^{2}\left(\mathscr{D}^{F}, \mathbb{C}\right) \cong \mathbb{C}^{3}$.

On the other hand, let $\pi: \mathscr{C} \rightarrow S$ be a family of genus $g$ compact Riemann surfaces and let $\omega_{\mathscr{C} / S}$ be the relative dualizing sheaf of $\pi$. Denote by $\lambda_{n}$ the determinant line bundle of $\omega_{\mathscr{C} / S}^{n}$ on $S$. Then, as observed by Mumford [Mu], the Grothendieck-Riemann-Roch theorem for the family $\pi$ gives the following relation between Chern classes:

$$
c_{1}\left(\lambda_{n}\right)=\left(6 n^{2}-6 n+1\right) c_{1}\left(\lambda_{1}\right) .
$$

One of the main objectives of the present paper is to explain the coincidence of $(0.1)$ and (0.2). In order to achieve this it is therefore of central importance to us to find a relationship between extensions of our Lie algebras and line bundles on moduli spaces.

Let us briefly introduce the moduli spaces involved in our construction. First of all the moduli space $\mathscr{M}_{g}$ of smooth curves of genus $g$, then the moduli space $\mathscr{M}_{g}^{\prime \prime}$ of triples $(C, p, v)$ when $C$ is a genus $g$ Riemann surface, $p$ a point on $C$, and $v$ a nonzero tangent vector to $C$ at $p$. We also consider the moduli space $\mathscr{F}_{h}^{\prime \prime}$ of quadruples $(C, p, v, L)$, where $L$ is a degree $h$ line bundle on $C$ and $(C, p, v) \in \mathscr{M}_{g}^{\prime \prime}$. 
Furthermore, we construct an infinite dimensional complex manifold $\hat{M}_{g}$ which is a moduli space of triples $(C, p, z)$, where $z$ is a local parameter at $p$. Finally, we construct another infinite dimensional complex manifold $\widehat{\mathscr{F}}_{h}$ parametrizing quintuples $(C, p, z, L,[\phi])$, where $C, p, z, L$ are as above, $\phi$ is a local trivialization of $L$ at $p$ and $[\phi]$ is the class of $\phi$ modulo non-zero multiplicative constants. Of course, we have natural projections

$$
\mathscr{M}_{g}^{\prime \prime} \rightarrow \mathscr{M}_{g}, \quad \hat{\mathscr{M}}_{g} \rightarrow \mathscr{M}_{g}^{\prime \prime}, \quad \hat{\mathscr{F}}_{h} \rightarrow \mathscr{\mathscr { F }}_{h}^{\prime \prime} .
$$

The first projection induces an isomorphism in second cohomology [actually, Harer, Ann. Math. 121, 215-249 (1985), has proven that $\mathscr{M}_{g}^{\prime \prime}$ has the same cohomology as $\mathscr{M}_{g}$ for $g$ large], the remaining two are homotopy equivalences.

By using the Kodaira-Spencer deformation theory on the infinite dimensional manifolds $\widehat{\mathscr{M}}_{g}$ and $\hat{\mathscr{F}}_{g-1}$ we get natural Lie algebra homomorphisms

$$
\begin{aligned}
\mathbf{d} & \rightarrow \operatorname{Vect}\left(\hat{\mathscr{M}}_{g}\right), \\
\mathscr{D} & \rightarrow \operatorname{Vect}\left(\hat{\mathscr{F}}_{g-1}\right),
\end{aligned}
$$

where $\mathbf{d}$ and $\mathscr{D}$ are suitable analytic analogues of $\mathbf{d}^{F}$ and $\mathscr{D}^{F}$ in which $\mathbf{d}^{F}$ and $\mathscr{D}^{F}$ are dense. The above homomorphisms have the property that for every $x \in \hat{\mathscr{M}}_{g}$ (respectively $\widehat{\mathscr{F}}_{g-1}$ ) the evaluation map

$$
p_{\mathbf{d}, x}: \mathbf{d} \rightarrow T_{x}\left(\hat{\mathscr{M}}_{g}\right) \quad\left(p_{\mathscr{D} . x}: \mathscr{D} \rightarrow T_{x}\left(\hat{\mathscr{F}}_{g-1}\right)\right)
$$

is surjective.

From this one gets that the tangent bundle $T\left(\hat{\mathscr{M}}_{g}\right)$ (respectively $\left.T\left(\hat{\mathscr{\mathscr { F }}}_{g-1}\right)\right)$ is canonically a quotient of the trivial bundle $\hat{\mathscr{M}}_{g} \times \mathbf{d}$ (respectively $\left.\hat{\mathscr{F}}_{g-1} \times \mathscr{D}\right)$. Similar results have been obtained in [BMS].

This allows us to define a canonical homomorphism

$$
\mu: H^{2}(\mathscr{D}) \rightarrow H^{1}\left(\Omega_{\hat{\mathscr{F}}_{\mathrm{g}-1}}^{1}\right)=\operatorname{Ext}^{1}\left(\mathscr{T}_{\hat{\mathscr{F}}_{\mathrm{g}-1}}, \mathcal{O}_{\hat{\mathscr{F}}_{g-1}}\right)
$$

(the case of $\hat{\mathscr{M}}_{g}$ is analogous). The definition of $\mu$ is as follows. Given a central extension

$$
0 \longrightarrow \mathbb{C} \longrightarrow \tilde{\mathscr{D}} \stackrel{\stackrel{o}{\longrightarrow}}{\longrightarrow} \longrightarrow 0
$$

we can lift canonically the inclusion

$$
\mathscr{D}_{x}=: \operatorname{Ker} p_{\mathscr{D}, x} \hookrightarrow \mathscr{D}
$$

to an inclusion

$$
\mathscr{D}_{x} \hookrightarrow \tilde{\mathscr{D}} .
$$

For this we use the following two facts:

$$
\mathscr{D}_{x}=\left[\mathscr{D}_{x}, \mathscr{D}_{x}\right] \text {, }
$$

ii) $\left.\varrho\right|_{\mathscr{D} x}$ is the trivial extension.

Using the inclusion $\mathscr{D}_{x} \hookrightarrow \mathscr{\mathscr { D }}$ we can construct an extension of the tangent bundle $T\left(\hat{\mathscr{F}}_{g-1}\right)$ whose fiber at $x$ is $\widetilde{\mathscr{D}} / \mathscr{D}_{x}$. Thus dualizing and passing to cohomology classes we get $\mu$. 
On the other hand, we have a natural homomorphism

$$
c: H^{1}\left(\mathcal{O}_{\mathscr{F}_{\mathfrak{g}-1}}^{*}\right) \rightarrow \operatorname{Ext}^{1}\left(\mathscr{T}_{\hat{\mathscr{F}}_{\mathfrak{g}-1}}, \mathcal{O}_{\hat{\mathscr{F}}_{\mathfrak{g}-1}}\right)=H^{1}\left(\Omega_{\hat{\mathscr{F}}_{\mathfrak{g}-1}}^{1}\right),
$$

associating to each line bundle $L$ the class of the extension

$$
0 \rightarrow \mathcal{O} \rightarrow \Sigma_{L} \rightarrow \mathscr{T} \rightarrow 0,
$$

where $\Sigma_{L}$ is the sheaf of differential operators of degree less than or equal to one on $L$. One of our results is that $c$ and $\mu$ have the same image.

Once this has been shown we get the following diagram:

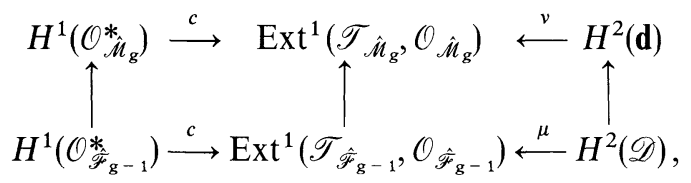

where the first two vertical arrows are induced by the canonical section $\hat{\mathscr{M}}_{g} \rightarrow \hat{\mathscr{F}}_{g-1}$, associating to a triple $(C, p, z)$ the quintuple $\left(C, p, z, \mathcal{O}((g-1) p),\left[z^{-g+1}\right]\right)$. Thus we obtain an explicit connection between the cohomology of $\mathscr{D}$ (respectively d) and line bundles on $\hat{\mathscr{F}}_{g-1}$ (respectively $\hat{\mathscr{M}}_{g}$ ). To see that $\mu$ and $c$ have the same image, we first notice that $\mathscr{\mathscr { F }}_{g-1}$ and $\mathscr{D}$ are acted on by automorphisms $\tau$ and $t$ defined by

$$
\begin{aligned}
\tau((C, p, z, L,[\phi])) & =\left(C, p, z, L \otimes \omega_{C}((2-2 g) p),\left[\phi z^{2 g-2} d z\right]\right), \\
t\left(a(z)+b(z) \frac{d}{d z}\right) & =a(z)+b^{\prime}(z)+(2 g-2) z^{-1} b(z)+b(z) \frac{d}{d z},
\end{aligned}
$$

which are related by the commutative diagram

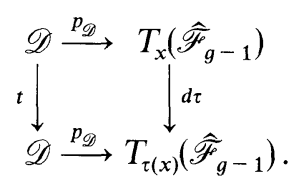

Using this diagram and the fact that $H^{2}(\mathscr{D})$ is a cyclic module over the group $\left\{t^{k}\right\}_{k \in \mathbb{Z}}$ with cyclic element $\psi_{0}=\phi_{0}^{*}(-\psi)$, we are reduced to show that $\mu\left(\psi_{0}\right)$ lies in the image of $c$. Indeed, we consider the divisor $\theta$ on $\widehat{\mathscr{F}}_{g-1}$ consisting of quintuples $(C, p, z, L,[\phi])$ with $L$ effective, and show that

$$
\mu\left(\psi_{0}\right)=c(\theta) .
$$

To prove this equality we use a global version of a construction due to Krichever and analyzed in [SW], giving an analytic map

$$
W: \widehat{\mathscr{F}}_{g-1} \rightarrow \operatorname{Gr}(H),
$$

where $\operatorname{Gr}(H)$ is a suitably defined infinite dimensional Grassmannian. We then have that $\mathcal{O}(-\theta)$ is the pullback, via $W$, of the determinant line bundle $\mathscr{L}$ on $\operatorname{Gr}(H)$.

On the other hand, we have the following commutative diagram

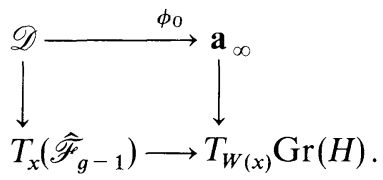


By an argument similar to the one above we associate to the extension of $\mathbf{a}_{\infty}$ given by the cocycle $\psi$ an extension of $\mathscr{T}_{\mathrm{Gr}(H)}$ by $\mathcal{O}_{\mathrm{Gr}(H)}$ which turns out to be the sheaf $\Sigma_{\mathscr{L}}$ of differential operators of degree less than or equal to 1 on $\mathscr{L}$. We can then pull back this extension to an extension of $\mathscr{T}_{\hat{\mathscr{F}}_{g-1}}$, which by functoriality is the sheaf of differential operators of degree less than or equal to one on $\mathcal{O}(-\theta)$. The above diagram then gives $(0.4)$.

Following diagram (0.3) and our analysis of the homomorphisms $t$ and $\tau$ we deduce that

$$
c\left(\lambda_{n}\right)=\left(6 n^{2}-6 n+1\right) c\left(\lambda_{1}\right) .
$$

In fact, by use of a result of Harer [H], and a generalization of it for $\mathscr{F}_{g-1}^{\prime \prime}$ which we explain in Sect. 5, we can then conclude:

Theorem. There are canonical homomorphisms, which are isomorphisms for $g \geqq 5$,

such that

$$
\begin{gathered}
v: H^{2}(\mathbf{d}) \rightarrow H^{2}\left(\mathscr{M}_{g}, \mathbb{C}\right), \\
\mu: H^{2}(\mathscr{D}) \rightarrow H^{2}\left(\mathscr{F}_{g-1}^{\prime \prime}, \mathbb{C}\right),
\end{gathered}
$$

(i) $\mu\left(\psi_{0}\right)=c_{1}(\theta)$.

(ii) The diagram

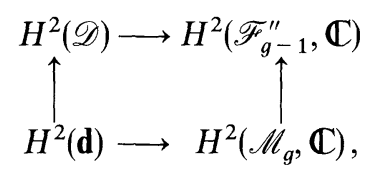

where the vertical arrows are induced by the obvious projections, commutes.

(iii) The diagram

commutes.

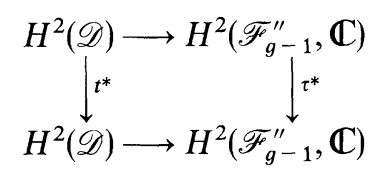

The paper is organized as follows. In Sect. 1I) we recall the basic facts about moduli spaces of curves; in Sect. 1II) we introduce a locally convex topological vector space $H$ which we believe gives the right setting for the study of the Krichever construction. We then introduce the infinite dimensional Grassmannian $\operatorname{Gr}(H)$ and its determinant line bundle by adapting to our situation the constructions given in [KP, SW, SP].

In Sect. 2 we compute the second cohomology of $\mathscr{D}$ and study the action of the automorphism $t$ on it.

In Sect. 3 we construct the infinite dimensional moduli spaces $\hat{\mathscr{M}}_{g}$ and $\hat{\mathscr{F}}_{g-1}$, define the global Krichever map and study its infinitesimal behaviour.

In Sect. 4 we give the above mentioned relation between line bundles on moduli spaces and cohomology of $\mathscr{D}$.

In Sect. 5 we compute $H^{2}\left(\mathscr{F}_{g-1}^{\prime \prime}\right)=H^{2}\left(\widehat{\mathscr{F}}_{g-1}\right)$, using results of $[\mathrm{H}]$.

In the Appendix (Sect. 6) we classify the degenerate and the unitary highest weight representations of the universal central extension of the Lie algebra $\mathscr{D}^{F}$.

Some of the results of this paper were quoted in [AGR]. 


\section{Notation and Preliminaries}

\section{I) Curves and Their Moduli}

For any family of smooth curves

$$
\pi: \mathscr{C} \rightarrow S,
$$

parametrized by an analytic space $S$, we shall denote by $\omega_{\mathscr{C} / S}$ the relative dualizing sheaf of $\pi$. We shall denote by $C_{s}$ the fiber $\pi^{-1}(s)$ over a point $s$ in $S$. Given a line bundle $\mathscr{L}$ on $\mathscr{C}$, we recall that one may define a determinant line bundle $\operatorname{det}_{\pi}(\mathscr{L})$ over $S$ as follows [MK]. One takes an auxiliary line bundle $M$ on $\mathscr{C}$ of very high relative degree. A global section $\sigma$ of $M$, which does not vanish identically on any fiber of $\pi$, gives an exact sequence

$$
0 \rightarrow \pi_{*} \mathscr{L} \rightarrow \pi_{*}(\mathscr{L} \otimes M)=E \rightarrow F=\pi_{*}\left(\left.\mathscr{L} \otimes M\right|_{\sigma=0}\right) \rightarrow R^{1} \pi_{*} \mathscr{L} \rightarrow 0 .
$$

As $E$ and $F$ are locally free one sets

$$
\operatorname{det}_{\pi}(\mathscr{L})=\left(\bigwedge^{\max } E\right) \otimes\left(\bigwedge^{\max } F\right)^{-1}
$$

and then checks that this definition does not depend on the choice of $M$ and $\sigma$. One then defines line bundles $\lambda_{n}$ on $S$ by setting

$$
\lambda_{n}(S)=\operatorname{det}_{\pi}\left(\omega_{\mathscr{C} / S}^{n}\right), \quad n \in \mathbb{Z} .
$$

Often, when no confusion will arise, we shall drop the $S$ and the $\pi$ in the notation and simply write det, $\omega$, and $\lambda_{n}$. The line bundle $\lambda_{1}$ is known as the Hodge bundle. For any integer $h$ we shall also consider the relative Picard variety

$$
\operatorname{Pic}^{h}(\pi) \rightarrow S
$$

whose fiber over $s \in S$ is $\mathrm{Pic}^{h}\left(C_{s}\right)$. Finally, associated to the relative Picard variety $\operatorname{Pic}^{g-1}(\pi)$ is a relative theta-divisor $\theta_{\pi}$, or simply $\theta$, which is a line bundle on $\operatorname{Pic}^{g-1}(\pi)$, whose restriction to $\operatorname{Pic}^{g-1}\left(C_{s}\right)$ is the theta-divisor $\mathcal{O}\left(\Theta_{s}\right)$ (for each $s$ ).

A family of pointed curves

$$
\mathscr{C} \underset{\sigma}{\stackrel{\pi}{\rightleftarrows}} S
$$

parametrized by $S$ is a family of curves $\pi$ equipped with a section $\sigma$ of $\pi$. When dealing with pointed curves we can define a canonical section of $p: \operatorname{Pic}^{h}(\pi) \rightarrow S$ :

canonical isomorphisms

$$
\begin{aligned}
\xi=\xi_{\sigma}: & \rightarrow \operatorname{Pic}^{h}(\pi), \\
s & \mapsto\left(s, \mathcal{O}_{C_{s}}(h \sigma)\right),
\end{aligned}
$$

$$
\begin{aligned}
\eta=\eta_{\sigma}^{h, k}: \operatorname{Pic}^{h}(\pi) & \rightarrow \operatorname{Pic}^{k}(\pi), \\
(s, L) & \mapsto\left(s, L \otimes \mathcal{O}_{C_{s}}((k-h) \sigma(s))\right),
\end{aligned}
$$

and, for $h=g-1$, a translation isomorphism

$$
\begin{aligned}
\tau=\tau_{2 g-2}: \operatorname{Pic}^{g-1}(\pi) & \rightarrow \operatorname{Pic}^{g-1}(\pi), \\
(s, L) & \mapsto\left(s, L \otimes \omega_{C_{s}}((2-2 g) \sigma(s))\right) .
\end{aligned}
$$


Consider the diagram

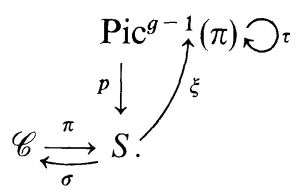

Consider the relative theta-divisor $\theta=\theta_{\pi}$ on $\operatorname{Pic}^{g-1}(\pi)$ and set

$$
\theta_{n \tau}=\tau^{* n} \theta, \quad n \in \mathbb{Z} .
$$

We have the following

(1.7) Lemma. $\xi^{*} \theta_{n \tau} \cong \lambda_{n}^{-1} \otimes \omega^{(2 n-1)^{2}}\left(\begin{array}{l}g \\ 2\end{array}\right)$, where $\lambda_{n}=\lambda_{n}(S)$ and $\omega=\sigma^{*} \omega_{\mathscr{C} / S} \cdot[$ Note that $(2 n-1)^{2}\left(\begin{array}{l}g \\ 2\end{array}\right) \operatorname{deg} \omega$ is equal to the number of Weierstrass points for $\omega^{n}$.

To give the straightforward proof of this isomorphism we recall the basic properties of Poincaré bundles.

Given the family of pointed curves (1.6), construct first the fiber product

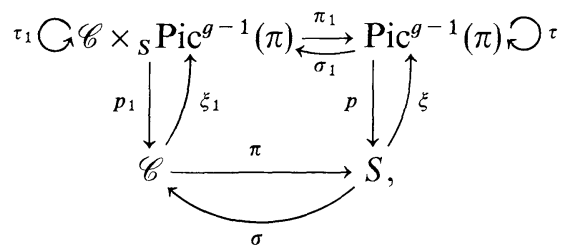

where $\pi_{1}, \sigma_{1}, p_{1}, \xi_{1}$ are the obvious maps and $\tau_{1}((q, L))=(q, \tau(L))$. A Poincare line bundle $\mathscr{L}_{\pi, \sigma}$, or simply $\mathscr{L}$ is a line bundle on $\mathscr{C} \times{ }_{S} \operatorname{Pic}^{g-1}(\pi)$ such that

$$
\begin{gathered}
\left.\mathscr{L}\right|_{C_{s} \times\{L\}} \cong L, \\
\sigma_{1}^{*} \mathscr{L}=p^{*} \sigma^{*}\left(\omega_{\mathscr{C} / S}\right)=\sigma_{1}^{*} p_{1}^{*}\left(\omega_{\mathscr{C} / S}\right) .
\end{gathered}
$$

It follows from the definition that

$$
\operatorname{det}_{\pi_{1}}(\mathscr{L})=\theta_{\pi}^{-1}
$$

and that

$$
\xi_{1}^{*} \tau_{1}^{* n}(\mathscr{L})=\omega_{\mathscr{C} / S}^{n}((2 n-1)(1-g) \Delta),
$$

where $\Delta$ is the image in $\mathscr{C}$ of the section $\sigma$. To prove the lemma we then notice that

$$
\begin{aligned}
\xi^{*} \theta_{n \tau}^{-1} & =\xi^{*} \tau^{* n} \theta^{-1}=\xi^{*} \tau^{* n} \operatorname{det}_{\pi_{1}}(\mathscr{L}) \\
& =\operatorname{det}_{\pi}\left(\xi_{1}^{*} \tau_{1}^{* n} \mathscr{L}\right)=\operatorname{det}_{\pi}\left(\omega_{\mathscr{C} / S}^{n}((2 n-1)(1-g) \Delta)\right) .
\end{aligned}
$$

On the other hand, one has

$$
\operatorname{det}_{\pi} \omega_{\mathscr{C} / S}^{n}(-N \Delta)=\lambda_{n} \otimes \omega^{-N n-\left(\begin{array}{l}
N \\
2
\end{array}\right)} .
$$

[This formula is proved by induction from the exact sequence

$$
0 \rightarrow \operatorname{det}_{\pi} \omega_{\mathscr{C} / S}^{n}(-N \Delta) \rightarrow \operatorname{det}_{\pi} \omega_{\mathscr{C} / S}^{n}(-(N-1) \Delta) \rightarrow \operatorname{det}_{\pi}\left(\omega_{\mathscr{C} / S}^{n}\left(-\left.(N-1) \Delta\right|_{\Delta}\right) \rightarrow 0,\right.
$$


by noticing that the third term of this sequence is isomorphic to $\omega^{n+N-1}$.] The lemma follows from (1.10) and (1.11). Q.E.D.

As a consequence we get the following relative version of the theorem of the square.

(1.12) Corollary. $\theta_{n \tau}=\theta_{(n-1) \tau} \otimes \theta_{\tau} \otimes \theta^{-1} \otimes p^{*}\left(\lambda_{n}^{-1} \otimes \lambda_{n-1} \otimes \omega^{8(n-1)}\left(\begin{array}{l}g \\ 2\end{array}\right)\right)$.

Proof. By the theorem of the square we have

$$
\theta_{n t}=\theta_{(n-1) \tau} \otimes \theta_{\tau} \otimes \theta^{-1} \otimes p^{*} \mathscr{L}
$$

for some line bundle $\mathscr{L}$ on $S$. To compute $\mathscr{L}$ apply $\xi^{*}$ to both sides in the above equality and use Lemma (1.7). Q.E.D.

We end this subsection by recalling some standard notation and introducing some terminology.

We denote by $\mathscr{M}_{g}$ the moduli space of curves of genus $g$. When $g>1$ we let $\mathscr{M} \subset \mathscr{M}_{g}$ be the open set corresponding to automorphism free curves. The open set $\mathscr{M}$ is the parameter space for a family of smooth genus $g$ curves

$$
\pi: \mathscr{M}^{(1)} \rightarrow \mathscr{M}
$$

having the property that the fiber of $\pi$ over a point $x \in \mathscr{M}$ is a smooth genus $g$ curve representing $x$.

A family of pointed curves with (non-zero) cotangent vector is a family of pointed curves

$$
\mathscr{C} \underset{\sigma}{\stackrel{\pi}{\rightleftarrows}} S
$$

together with a nowhere vanishing section $\tau$ of $\sigma^{*}\left(\omega_{\mathscr{C} / S}\right)$, i.e. a trivialization of $\sigma^{*}\left(\omega_{\mathscr{C} / S}\right)$. Consider the functor

$$
\begin{aligned}
\left\{\begin{array}{l}
\text { Analytic } \\
\text { spaces }
\end{array}\right\} & \rightarrow\{\text { sets }\}, \\
\qquad & \leadsto\left\{\begin{array}{l}
\text { Families of pointed curves } \\
\text { with a cotangent vector } \\
\text { parametrized by } S
\end{array}\right\}
\end{aligned}
$$

Since there is no non-trivial automorphism of a Riemann surface of genus $g>0$ fixing a point and a non-zero (co)-tangent vector, one can see that this functor is represented by a smooth quasi-projective variety $\mathscr{M}_{g}^{\prime \prime}$ of dimension $3 g-1$ (here $g>1$ ). This variety is equipped with a universal family of pointed curves with cotangent vector

$$
\mathscr{C}^{\prime \prime} \underset{\sigma^{\prime \prime}}{\stackrel{\pi^{\prime \prime}}{\rightleftarrows}} \mathscr{M}_{g}^{\prime \prime}, \quad \tau \text { a nowhere vanishing section of } \sigma^{*}\left(\omega_{\mathscr{C}^{\prime \prime} / \mathcal{M}_{g}^{\prime \prime}}\right) .
$$

Clearly, the open set $\mathscr{M}^{\prime \prime}$ of $\mathscr{M}_{g}^{\prime \prime}$ consisting of triples $(C, p, v)$, where $C$ is automorphism free, can be identified with the $\mathbb{C}^{\times}$-bundle

$$
\omega_{\mathscr{M}^{(1)} / \mathscr{M}}-\{0 \text {-section }\} \rightarrow \mathscr{M}^{(1)}
$$




\section{II) A Locally Convex Topological Vector Space and Its Associated Grassmann Manifold}

In this subsection we shall introduce an infinite dimensional Grassmann manifold. This Grassmannian, as well as the infinite dimensional manifolds that we shall construct in the sequel, will be modelled on the topological vector spaces we presently describe.

For $\varepsilon>0$ let $D_{\varepsilon}=\{z \in \mathbb{C}:|z|<\varepsilon\}$ and $\dot{D}_{\varepsilon}=D_{\varepsilon}-\{0\}$. Let $H$ be the complex vector space of all germs at 0 of complex valued holomorphic functions possibly singular at 0 :

$$
H=\lim _{\varepsilon \rightarrow 0} \mathcal{O}\left(\dot{D_{\varepsilon}}\right) .
$$

Let $\hat{\mathbb{C}}$ denote the Riemann sphere. Let $H_{-}$be the space of all holomorphic functions on $\hat{\mathbb{C}}-\{0\}$ which vanish at $\infty$, and let $H_{+}$be the space of germs of holomorphic functions at 0 :

$$
H_{+}=\lim _{\varepsilon \rightarrow 0} \mathcal{O}\left(D_{\varepsilon}\right) \quad(=\mathbb{C}\{z\}) .
$$

We then have a canonical decomposition

$$
H=H_{+} \oplus H_{-} .
$$

This follows from the elementary

(1.15) Lemma. There is a canonical decomposition

$$
\mathcal{O}\left(\dot{D}_{\varepsilon}\right)=H_{-} \oplus \mathcal{O}\left(D_{\varepsilon}\right) .
$$

Proof. Computing the cohomology of $\mathcal{O}_{\hat{\mathbb{C}}}$ by using the covering $\left\{D_{\varepsilon}, \hat{\mathbb{C}}-\{0\}\right\}$ gives the exact sequence

$$
0 \rightarrow H^{0}(\widehat{\mathbb{C}}, \mathcal{O})=\mathbb{C} \rightarrow H^{0}(\widehat{\mathbb{C}}-\{0\}, \mathcal{O}) \oplus H^{0}\left(D_{\varepsilon}, \mathcal{O}\right) \rightarrow H^{0}\left(\dot{D}_{\varepsilon}, \mathcal{O}\right) \rightarrow H^{1}(\widehat{\mathbb{C}}, \mathcal{O})=0 .
$$

We then get the exact sequence

$$
0 \longrightarrow \mathbb{C} \stackrel{\phi}{\longrightarrow} H_{-} \oplus \mathbb{C} \oplus \mathcal{O}\left(D_{\varepsilon}\right) \longrightarrow \mathcal{O}\left(\dot{D}_{\varepsilon}\right) \longrightarrow 0,
$$

where $\phi(a)=(0, a, a)$, proving the lemma. Q.E.D.

If a function $f$ is defined and continuous on the circle $|z|=c$, we may define

$$
\|f\|_{c}=\max _{|z|=c}|f(z)| \text {. }
$$

This gives us a norm \|\|$_{c}$, for all $c>0$, on $H_{-}$giving $H_{-}$the usual Fréchet topology. Given an open subset $Y \subset \mathbb{C}^{n}$, denote by $H(Y)$ the space of all continuous functions on $\bar{Y}$ which are holomorphic on $Y$. With the norm \|\|$_{\varepsilon}, H\left(D_{\varepsilon}\right)$ is a Banach space. We have $H_{+}=\lim _{\varepsilon \rightarrow 0} H\left(D_{\varepsilon}\right)$, and we give $H_{+}$the direct limit topology. Thus $H$ becomes a locally convex topological space (with the product topology). Note also that $H_{+}$and $H_{-}$are (topological) dual of each other and that polynomials are dense in both spaces.

Given topological vector spaces $U$ and $V$ we denote by $M(U, V)$ the space of all continuous linear maps from $U$ to $V$. We are now going to describe $M\left(H_{+}, H_{-}\right)$ 
and $M\left(H_{-}, H_{+}\right)$. Given a function $f(z, w)$ holomorphic in a neighbourhood of $(0,0)$, we associate to it the operator

$$
\begin{aligned}
T_{f}: H_{-} & \rightarrow H_{+}, \\
h\left(z^{-1}\right) & \mapsto \operatorname{Res}_{w=0} f(z, w) h\left(w^{-1}\right) .
\end{aligned}
$$

Similarly, given a function $g\left(z^{-1}, w^{-1}\right)$ holomorphic on $(\widehat{\mathbb{C}}-\{0\}) \times(\widehat{\mathbb{C}}-\{0\})$ and vanishing on $(\{\infty\} \times \widehat{\mathbb{C}}) \cup(\hat{\mathbb{C}} \times\{\infty\})$, we associate to it the operator

$$
\begin{aligned}
T_{g}: H_{+} & \rightarrow H_{-}, \\
h(z) & \mapsto \operatorname{Res}_{w=0} g\left(z^{-1}, w^{-1}\right) h(w) .
\end{aligned}
$$

It is easy to verify that these operators are continuous and that the space $M\left(H_{-}, H_{+}\right)$(respectively $M\left(H_{+}, H_{-}\right)$) consist of operators of the form $T_{f}$ (respectively $T_{g}$ ) $[\mathrm{R}]$. Setting then

$$
\begin{aligned}
& H_{+-}=\underset{\longrightarrow}{\lim } H\left(D_{\varepsilon, \varepsilon}\right) \quad(=: \mathbb{C}\{z, w\}), \\
& \left.H_{-+}=\left\{g\left(z^{-1}, w^{-1}\right) \in H(\hat{\mathbb{C}}-\{0\}) \times(\hat{\mathbb{C}}-\{0\})\right): g\left(\infty, w^{-1}\right)=g\left(z^{-1}, \infty\right)=0\right\},
\end{aligned}
$$

we get identifications

$$
M\left(H_{-}, H_{+}\right) \cong H_{+-}, \quad M\left(H_{+}, H_{-}\right) \cong H_{-+} .
$$

We think of $H_{+_{-}}$as equipped with the direct limit topology and $H_{-+}$with the usual Fréchet topology. We then observe that, in these topologies, the maps of finite rank are dense since polynomials in two variables are dense in both spaces. Finally, we notice that also the spaces $M\left(H_{-}, H_{-}\right)$and $M\left(H_{+}, H_{+}\right)$may be explicitly described, namely

$$
\begin{aligned}
M\left(H_{-}, H_{-}\right) \cong H_{--}= & \left\{f\left(z^{-1}, w\right): f\right. \text { holomorphic in an open set } \\
& \text { of }(\hat{\mathbb{C}}-\{0\}) \times \mathbb{C} \text { containing }(\hat{\mathbb{C}}-\{0\}) \times\{0\} \\
& \text { and vanishing on }\{\infty\} \times \mathbb{C}\}, \\
M\left(H_{+}, H_{+}\right) \cong H_{++}= & \left\{f\left(z, w^{-1}\right): f\right. \text { holomorphic in an open set } \\
& \text { of } \mathbb{C} \times(\hat{\mathbb{C}}-\{0\}) \text { containing }\{0\} \times(\hat{\mathbb{C}}-\{0\}) \\
& \text { and vanishing on } \mathbb{C} \times\{\infty\}\} .
\end{aligned}
$$

Of course, $H_{++}$is isomorphic to $H_{--}$by exchanging $z$ and $w$.

We shall denote by $\mathbf{a}_{\infty}$ the Lie algebra of all continuous endomorphisms of $H=H_{-} \oplus H_{+}$. Every element $a$ of $\mathbf{a}_{\infty}$ can be written in the form

$$
a=\left(\begin{array}{ll}
a_{++} & a_{+-} \\
a_{-+} & a_{--}
\end{array}\right),
$$

where $a_{++} \in M\left(H_{+}, H_{+}\right)$and so on. By our previous descriptions every element of $\mathbf{a}_{\infty}$ can be interpreted as an operator $T_{f}: H \rightarrow H$, where

$$
f=f_{--}\left(z^{-1}, w\right)+f_{-+}\left(z^{-1}, w^{-1}\right)+f_{+-}(z, w)+f_{++}\left(z, w^{-1}\right),
$$


where $f_{++} \in H_{++}$and so on. Explicitly

$$
\begin{aligned}
T_{f}\left(h_{-}+h_{+}\right)= & \operatorname{Res}_{w=0}\left(f_{--}\left(z^{-1}, w\right) h_{-}\left(w^{-1}\right)+f_{-+}\left(z^{-1}, w^{-1}\right) h_{+}(w)\right. \\
& \left.\times f_{+-}(z, w) h_{-}\left(w^{-1}\right)+f_{++}\left(z, w^{-1}\right) h_{+}(w)\right) .
\end{aligned}
$$

(Notice that all terms make perfect sense.)

We now want to make a few remarks about composition and traces. First of all, given two operators $T_{f}$ and $T_{g}$ in $\mathbf{a}_{\infty}$, we have

where $f * g$ is the convolution

$$
T_{f} \circ T_{g}=T_{f * g}
$$

$$
(f * g)(z, w)=\operatorname{Res}_{t=0} f(z, t) g(t, w) .
$$

Secondly, notice that given operators

$$
T_{f_{+-}}: H_{-} \rightarrow H_{+}, \quad T_{f_{-+}}: H_{+} \rightarrow H_{-}
$$

the compositions $T_{f_{+-}} \circ T_{f_{-+}}$and $T_{f_{-+}} \circ T_{f_{+-}}$are both of trace class and their trace is given by the formula

$$
\operatorname{Res}_{z=w=0} f_{-+}\left(z^{-1}, w^{-1}\right) f_{+-}(w, z)=\operatorname{Res}_{z=w=0} f_{+-}(w, z) f_{-+}\left(z^{-1}, w^{-1}\right) .
$$

Using this we immediately get that $\mathbf{a}_{\infty}$ carries a cocycle

$$
\psi(a, b)=\operatorname{Trace}\left(b_{-+} a_{+-}-a_{-+} b_{+-}\right) .
$$

Finally, we let $A_{\infty}$ be the set of all continuous invertible operators $a$ on $H$ such that $a_{-}$and $a_{++}$are Fredholm of index zero. One checks that this is a group under multiplication which is the identity component of the group $M(H, H)^{\times}$.

We now define the Grassmannian $\operatorname{Gr}(H)$ as the set of closed subspaces $W$ of $H$ such that $p_{-}: W \rightarrow H_{-}$is Fredholm of index 0 . Here $p_{-}: H \rightarrow H_{-}$denotes the projection operator on $H_{-}$. We shall give $\operatorname{Gr}(H)$ the structure of a complex manifold modelled on the space $M\left(H_{-}, H_{+}\right)$. In order to do that introduce the set $\Sigma$ of sequences of integers $S=\left\{s_{1}>s_{2}>\ldots\right\}$ such that $s_{n}=-n$ for $n \gg 0$, and the associated subspaces $H_{S}$ and $H_{S}^{+}$which are the closure of the linear spans of the sets $\left\{z^{s_{j}}: s_{j} \in S\right.$ (respectively $\left.\left.s_{j} \notin S\right)\right\}$ in $H$. Note that $H_{S} \in \operatorname{Gr}(H)$.

(1.18) Lemma (see $[\mathrm{PS}])$. Given $W \in \mathrm{Gr}(H)$ there exists a sequence $S \in \Sigma$ such that the projection $p_{S}: W \rightarrow H_{S}$ is an isomorphism.

Proof. Choose a basis of Ker $\left.p_{-}\right|_{W}$ of the form: $z^{i_{1}}+$ higher order terms, $z^{i_{2}}+$ higher order terms, $\ldots, z^{i_{s}}+$ higher order terms, where $i_{1}>i_{2}>\ldots>i_{s} \geqq 0$. Choose a basis of $H_{-} \bmod p_{-}(W)$ of the form: $z^{j_{1}}, \ldots, z^{i_{s}}$ such that $0>j_{1}>j_{2}>\ldots>j_{s}$. Then $S=\left\{i_{1}, i_{2}, \ldots, i_{s},-1, \ldots, \hat{j_{1}}, \ldots, \hat{j_{s}}, \ldots\right\}$ is the required sequence. Q.E.D.

For $S \in \Sigma$ set

$$
\operatorname{Gr}^{S}(H)=\left\{W \in \operatorname{Gr}(H) \mid p_{S}: W \rightarrow H_{S} \text { is an isomorphism }\right\} .
$$

It is clear that we have a canonical identification

$$
\mathrm{Gr}^{5}(H) \cong M\left(H_{-}, H_{+}\right)
$$


obtained by taking graphs, and by identifying $H_{-}$with $H_{S}$ and $H_{+}$with $H_{S}^{+}$in the obvious way. One easily checks that this gives $\operatorname{Gr}(H)$ the structure of an infinite dimensional complex manifold. We remark that the group $A_{\infty}$ acts transitively on $\operatorname{Gr}(H)$ making it a homogeneous space.

We now turn to the construction of the determinant line bundle $\mathscr{L}$ on $\operatorname{Gr}(H)$. For $W \in \operatorname{Gr}(H)$ let $K_{W}=\left.\operatorname{Ker} p_{-}\right|_{W}$ and $C_{W}=\left.\operatorname{Coker} p_{-}\right|_{W}$. Set

$$
\mathscr{L}_{W}=\bigwedge^{\max } K_{W} \otimes \bigwedge^{\max }\left(C_{W}\right)^{*} .
$$

(1.20) Lemma. Let $W \in \mathrm{Gr}^{S}(H)$; then we have a canonical isomorphism between $\mathscr{L}_{W}$ and $\mathscr{L}_{H_{S}}$.

Proof. Notice that $p_{S}\left(K_{W}\right) \subset K_{H_{S}}$. Since $p_{S}: W \rightarrow H_{S}$ is an isomorphism, we can identify $K_{H_{S}}$ with a subspace of $W$. On the other hand, we can identify $C_{H_{S}}$ with the subspace of $H_{-}$spanned by the elements $z^{i}, i<0, i \notin S$. This gives us the following sequence, whose exactness one easily proves:

$$
0 \rightarrow K_{W} \rightarrow K_{H_{S}} \rightarrow C_{H_{S}} \rightarrow C_{W} \rightarrow 0,
$$

and the lemma is proved. Q.E.D.

Using this lemma we can canonically identify the restriction of $\mathscr{L}$ to $\mathrm{Gr}^{\mathrm{S}}(H)$ with $\mathrm{Gr}^{\mathrm{S}}(H) \times \mathscr{L}_{H_{S}}$, getting a trivialization of $\mathscr{L}$ on the charts $\mathrm{Gr}^{S}(H)$. This gives $\mathscr{L}$ the structure of a holomorphic line bundle on $\operatorname{Gr}(H)$.

Set now

$$
\mathrm{Gr}_{0}^{(m)}=\left\{W \in \mathrm{Gr}(H) \mid z^{m} H_{-} \supset W \supset z^{-m} H_{-}\right\} .
$$

Note that we have a canonical isomorphism

$$
\mathrm{Gr}_{0}^{(m)}=\mathrm{Gr}(2 m, m)
$$

Set

$$
\mathrm{Gr}_{0}=\bigcup_{m \geqq 0} \mathrm{Gr}_{0}^{(m)} \subset \mathrm{Gr}(H) .
$$

Remark that $\mathrm{Gr}_{0} \cap \mathrm{Gr}^{S}(H)$ consists, under the canonical identification of $\mathrm{Gr}_{H}^{S}$ with $M\left(H_{-}, H_{+}\right)$, of operators of the form $T_{f}$, where $f$ is a polynomial in $z$ and $w$. Thus we obtain the following

(1.21) Lemma. $\mathrm{Gr}_{0}$ is dense in $\mathrm{Gr}(H)$. In particular, every holomorphic function on $\mathrm{Gr}(H)$ is constant.

We are now going to define a central extension $\widetilde{A}_{\infty}$ of $A_{\infty}$ which will act on the determinant bundle $\mathscr{L}$. First, we consider the subspace $J_{0}$ of $M\left(H_{-}, H_{-}\right)$ spanned by those continuous maps which factor through $H_{+}$, i.e. the maps of the form $T_{f_{-+}}{ }^{\circ} T_{f_{+}-}$. It is clear that $J_{0}$ is an ideal. We then let $J$ be the subgroup of $G L\left(H_{-}\right)$consisting of the invertible maps of the form $1+j$ with $j \in J_{0}$. As we remarked in (1.16) the elements of $J_{0}$ are of trace class, so that the elements in $J$ have a determinant. The extension $\widetilde{A}_{\infty}$ of $A_{\infty}$ is defined as follows. Let $\mathscr{E}$ be the group

$$
\mathscr{E}=\left\{(a, q): a \in A_{\infty}, q \in G L\left(H_{-}\right), a_{--}-q \in J_{0}\right\}
$$


[to show that $\mathscr{E}$ is indeed a group, notice that given $(a, q)$ and $\left(a^{\prime}, q^{\prime}\right)$ in $\mathscr{E}$, then $\left(a a^{\prime}\right)_{--}=a_{--} a_{--_{-}}^{\prime}+a_{-+} a_{+-}^{\prime}$ and that $a_{--} a_{--_{-}}^{\prime}+a_{-+} a_{+-}^{\prime}-q q^{\prime}=\left(a_{--}-q\right) a_{--}^{\prime}$ $+q\left(a_{--}^{\prime}-q^{\prime}\right)+a_{-+} a_{+-}^{\prime}$ belongs to $\left.J_{0}\right]$. Consider the homomorphism

$$
\operatorname{det}: J \rightarrow \mathbb{C}^{*},
$$

let $J_{1}$ be its kernel, and set

$$
\tilde{A}_{\infty}=\mathscr{E} / J_{1},
$$

where $J_{1}$ is thought of as the subgroup

$$
\left\{(1, q), q \in J_{1}\right\} \subset \mathscr{E} .
$$

Clearly, $\tilde{A}_{\infty}$ is a central extension of $A_{\infty}$ by $\mathbb{C}^{\times}$. We leave it to the reader to verify (as in [PS, p. 89]) that the Lie algebra of $\tilde{A}_{\infty}$ is $\tilde{\mathbf{a}}_{\infty}=\mathbf{a}_{\infty} \oplus \mathbb{C}$ defined by the cocycle $\psi$.

We are now going to define an action of $\tilde{A}_{\infty}$ on $\mathscr{L}$. To do this we need some preparation. Given $W \in \operatorname{Gr}(H)$ let us define an admissible isomorphism to be an isomorphism

$$
w: H_{-} \rightarrow W,
$$

such that $p_{-} w$ is of the form $1+j$ with $j \in J_{0}$. We remark that given two admissible isomorphisms for $W$, say $w$ and $w^{\prime}$, then $w^{\prime-1} w \in J$. To see that there exists an admissible isomorphism for any given $W$, we can use Lemma (1.18) and choose a sequence $S \in \Sigma$ such that $W \in \operatorname{Gr}^{S}(H)$. We then get, by composition, an isomorphism

$$
\begin{gathered}
W \rightarrow H_{S} \rightarrow H_{-}, \\
z^{s_{i}} \mapsto z^{i}
\end{gathered}
$$

whose inverse $w$ is easily seen to be admissible. In fact, for such a $w$ we can choose an integer $n$ such that, in the decomposition $H_{-}=H_{\geqq-n} \oplus H_{<-n}$, where

$$
H_{\geqq-n}=\left\{\sum_{i=-1}^{-n} a_{i} z^{-i}\right\}, \quad H_{<-n}=\left\{\sum_{i<-n} b_{i} z^{-i}\right\},
$$

the matrix of $p_{-} w$ is of the form

$$
\left(\begin{array}{ll}
A & B \\
0 & I
\end{array}\right)
$$

In particular, the determinant of $p_{-} w$ is the determinant of the $n \times n$ matrix $A$. Furthermore, $w$ gives us a way of identifying $K_{W}$ with $\operatorname{Ker} p_{-} w$ and $C_{W}$ with Coker $p_{-} w$. We then get the exact sequence

$$
0 \longrightarrow K_{W} \longrightarrow H_{\geqq-n} \stackrel{A}{\longrightarrow} H_{\geqq-n} \longrightarrow C_{W} \longrightarrow 0
$$

which induces an identification

$$
h_{w}: \mathbb{C} \rightarrow \mathscr{L}_{W} .
$$

Suppose now given another admissible basis $w^{\prime}$ for $W$. Write $w^{\prime}=w q, q \in J$, then $h_{w^{\prime}}=h_{w} \operatorname{det} q$. We can finally define an action of $\tilde{A}_{\infty}$ on $\mathscr{L}$ by setting

$$
(A, q) \cdot(W, \lambda)=\left(A W, h_{A w q^{-1}} \circ h_{w^{-1}}(\lambda)\right)
$$


for any $(A, q) \in \mathscr{E}, W \in \operatorname{Gr}(H), \lambda \in \mathscr{L}_{W}$ and for any choice of an admissible isomorphism $w$. We leave it to the reader to verify that $A w q^{-1}$ is admissible, that the action does not depend on the choice of $w$ and that it factors though the quotient homomorphism $\mathscr{E} \rightarrow \widetilde{A}_{\infty}$.

\section{The Lie Algebra of Differential Operators of Order Less than or Equal to 1 in One Variable}

Let $H$ be the topological vector space considered in the preceding section. We let $\partial$ be the operator on $H$ defined by $\partial f=\frac{d f}{d z}=f^{\prime}$. It is easy, but important, to check that $\partial$ is a continuous operator on $H$. Consider the Lie algebra $\mathscr{D}=H \partial+H$ and the subalgebra $\mathbf{d} \subset \mathscr{D}, \mathbf{d}=H \partial$, with obvious Lie bracket. We shall denote by $H^{*}(\mathbf{g})$ the continuous Lie algebra cohomology of the topological Lie algebra $\mathbf{g}$.

(2.1) Proposition. 1) $H^{1}(\mathscr{D})=H^{1}(\mathbf{d})=0$.

2) $H^{2}(\mathbf{d}) \cong \mathbb{C}$ and is generated by the cohomology class of the cocycle

$$
\alpha(f \partial, g \partial)=\operatorname{Res}_{z=0} f d g^{\prime \prime} .
$$

3) $H^{2}(\mathscr{D}) \cong \mathbb{C}^{3}$ and is generated by the cohomology classes of the cocycles

$$
\left.\begin{array}{l}
\alpha_{1}\left(f_{1} \partial+g_{1}, f_{2} \partial+g_{2}\right)=\operatorname{Res}_{z=0} f_{1} d f_{2}^{\prime \prime} \\
\alpha_{2}\left(f_{1} \partial+g_{1}, f_{2} \partial+g_{2}\right)=\operatorname{Res}_{z=0}\left(f_{1} d g_{2}^{\prime}-f_{2} d g_{1}^{\prime}\right) \\
\alpha_{3}\left(f_{1} \partial+g_{1}, f_{2} \partial+g_{2}\right)=\operatorname{Res}_{z=0} g_{1} d g_{2}
\end{array}\right\} .
$$

Proof. Let $H^{F}=\mathbb{C}\left[z^{-1}, z\right] \subset H$. We have already noticed that $H^{F}$ is dense in $H$. So $\mathscr{D}^{F}=H^{F} \partial+H^{F}$ and $\mathbf{d}^{F}=H^{F} \partial$ are dense in $\mathscr{D}$ and $\mathbf{d}$, respectively. Let $d_{n}=z^{n+1} \partial$, $e_{n}=z^{n}$. The $d_{n}$ 's form a basis of $\mathbf{d}^{F}$ and the $d_{n}$ 's and $e_{n}$ 's a basis for $\mathscr{D}^{F}$. We have the following relations:

$$
\left[d_{n}, d_{m}\right]=(m-n) d_{n+m}, \quad\left[d_{n}, e_{m}\right]=m e_{n+m}, \quad\left[e_{n}, e_{m}\right]=0 .
$$

From this it is immediate to verify that $\mathscr{D}^{F}=\left[\mathscr{D}^{F}, \mathscr{D}^{F}\right], \mathbf{d}^{F}=\left[\mathbf{d}^{F}, \mathbf{d}^{F}\right]$. Using this and the fact that $\mathscr{D}^{F}$ (respectively $\mathbf{d}^{F}$ ) is dense in $\mathscr{D}$ (respectively $\mathbf{d}$ ), 1) follows. To show 2 ), we let $\alpha$ be a two-cocycle for $\mathscr{D}^{F}$ and we set

$$
\alpha_{n, m}=\alpha\left(d_{n}, d_{m}\right), \quad \alpha_{n}^{m}=\alpha\left(d_{n}, e_{m}\right), \quad \alpha^{n, m}=\alpha\left(e_{n}, e_{m}\right) .
$$

Looking at $\alpha\left(\left[d_{h}, e_{m}\right], e_{n}\right), \alpha\left(\left[d_{h}, d_{n}\right], e_{m}\right), \alpha\left(\left[d_{h}, d_{n}\right], d_{m}\right)$, using the relations (2.4) and the cocycle rule, we get the following

$$
\begin{aligned}
n \alpha^{h+n, m} & =m \alpha^{h+m, n} \\
(n-h) \alpha_{h+n}^{m} & =m\left(\alpha_{h}^{n+m}-\alpha_{n}^{h+m}\right) . \\
(n-h) \alpha_{h+n, m}+(m-n) \alpha_{n+m, h}+(h-m) \alpha_{h+m, n} & =0 .
\end{aligned}
$$

The first of these relations immediately implies

$$
\alpha^{n, m}=m \delta_{n,-m} \alpha^{-1,1}, \quad \forall n, m \in \mathbb{Z} .
$$


As for the second, setting $m=0$ we deduce that

$$
\alpha_{s}^{0}=0, \quad \forall s \in \mathbb{Z} .
$$

On the other hand, setting $h=0$, we get

$$
(n+m) \alpha_{n}^{m}=m \alpha_{0}^{n+m}, \quad \forall n, m \in \mathbb{Z},
$$

and setting $m=-h-n$, we get:

$$
(n-h) \alpha_{h+n}^{-h-n}=-(n+h)\left(\alpha_{h}^{-h}-\alpha_{n}^{-n}\right), \quad \forall n, h \in \mathbb{Z} .
$$

Finally, the last of our relations gives, setting $h=0$,

$$
(m-n) \alpha_{n+m, 0}+(n+m) \alpha_{n, m}=0, \quad \forall n, m \in \mathbb{Z},
$$

and setting $h=-n-m$, we get:

$$
(2 n+m) \alpha_{-m, m}+(m-n) \alpha_{n+m,-n-m}-(2 m+n) \alpha_{-n, n}=0, \quad \forall n, m \in \mathbb{Z} .
$$

Thus a general cocycle is completely determined by choosing values of $\alpha^{-1.1}, \alpha_{1}^{-1}$, $\alpha_{2}^{-2}, \alpha_{1,-1}, \alpha_{2,-2}, \alpha_{0}^{h}, \alpha_{h, 0}, \forall h \in \mathbb{Z}, h \neq 0$ [in fact, $\alpha^{-1,1}$ determines $\alpha^{n, m}$ via (2.7), $\alpha_{0}^{h}$ determines $\alpha_{n}^{m}, n+m \neq 0$, via (2.9), $\alpha_{1}^{-1}$ and $\alpha_{2}^{-2}$ determine $\alpha_{n}^{-n}$ via (2.10), $\alpha_{h, 0}$ determines $\alpha_{n, m}, n+m \neq 0$ via (2.11), $\alpha_{1,-1}$ and $\alpha_{2,-2}$ determine $\alpha_{n,-n}$ via (2.12)]. For example:

$$
\begin{gathered}
\alpha_{n, m}=\frac{m-n}{n+m} \alpha_{n+m, 0}, \quad n+m \neq 0, \\
\alpha_{n,-n}=-\frac{n\left(n^{2}-4\right)}{3} \alpha_{1,-1}+\frac{n^{3}-n}{6} \alpha_{2,-2} .
\end{gathered}
$$

By a straightforward computation one can then see that a general 2-cocycle for $\mathscr{D}$ is of the form

$$
\begin{aligned}
\alpha\left(f_{1} \partial+g_{1}, f_{2} \partial+g_{2}\right)= & \underset{z=0}{\operatorname{Res}}\left[a_{1} f_{1} d f_{2}^{\prime \prime}+a_{2}\left(f_{1} d g_{2}^{\prime}-f_{2} d g_{1}^{\prime}\right)\right. \\
& +a_{3} g_{1} d g_{2}+s(z)\left(f_{1} d f_{2}-f_{2} d f_{1}\right) \\
& \left.+t(z)\left(f_{1} d g_{2}-f_{2} d g_{1}\right)\right],
\end{aligned}
$$

where $a_{1}, a_{2}, a_{3} \in \mathbb{C}$ and $s(z), t(z) \in H$. Of course, the $a_{i}$ 's and the coefficients of $s$ and $t$ are linked to the constants $\alpha^{-1,1}, \alpha_{1}^{-1}, \alpha_{2}^{-2}, \alpha_{1,-1}, \alpha_{2,-2}, \alpha_{0}^{h}, \alpha_{h, 0}$. For example,

$$
a_{2}=\frac{1}{6}\left(2 \alpha_{1,-1}-\alpha_{2,-2}\right), \quad s_{-2}=-\frac{1}{2} d_{1,-1}, \quad s_{h-2}=-\frac{1}{h} \alpha_{-h, 0}, \quad h \neq 0,
$$

where $s(z)=\Sigma s_{n} z^{n}$. Notice also that all cocycles of the form $s(z)\left(f_{1} d f_{2}-f_{2} d f_{1}\right)$, $t(z)\left(f_{1} d g_{2}-f_{2} d g_{1}\right)$ are coboundaries. Using this our claim for $\mathscr{D}$ follows immediately. As far as $\mathbf{d}$ is concerned the claim in this case follows immediately from the above. Q.E.D.

Let us now consider the isomorphism

$$
t_{s}: \mathscr{D} \rightarrow \mathscr{D},
$$


defined by

$$
t_{s}(f \partial+g)=f \partial+g+f^{\prime}+s \frac{f}{z}, \quad s \in \mathbb{C} .
$$

We want to compute the action of the induced isomorphism

$$
t_{s}^{*}: H^{2}(\mathscr{D}) \rightarrow H^{2}(\mathscr{D}) \text {. }
$$

(2.16) Lemma. For any $s$ and $s^{\prime}, t_{s}^{*}=t_{s^{\prime}}^{*}$

Proof. It clearly suffices to show that the automorphism

$$
\sigma_{s}: \mathscr{D} \rightarrow \mathscr{D}
$$

defined by $\sigma_{s}(f \partial+g)=f \partial+g+s \frac{f}{z}$ induces the identity on $H^{2}(\mathscr{D})$. To see this notice that:

$$
\begin{aligned}
& \sigma_{s}^{*}\left(\alpha_{1}\right)=\alpha_{1} \\
& \sigma_{s}^{*}\left(\alpha_{2}\right)\left(f_{1} \partial+g_{1}, f_{2} \partial+g_{2}\right)=\operatorname{Res}_{z=0}\left[f_{1} d\left(\frac{s f_{2}}{z}+g_{2}\right)^{\prime}-f_{2} d\left(\frac{s f_{1}}{z}+g_{1}\right)^{\prime}\right] \\
& =\operatorname{Res}_{z=0}\left(f_{1} d g_{2}^{\prime}-f_{2} d g_{1}^{\prime}\right)+\underset{z=0}{\operatorname{Res}}-s z^{2}\left(f_{1} d f_{2}-f_{2} d f_{1}\right) \\
& +\operatorname{Res}_{z=0}\left(f_{1} d\left(\frac{f_{2}^{\prime}}{z}\right)-f_{2} d\left(\frac{f_{1}^{\prime}}{z}\right)\right) \\
& =\alpha_{2}+\underset{z=0}{\operatorname{Res}}-s z^{2}\left(f_{1} d f_{2}-f_{2} d f_{1}\right) \sim \alpha_{2},
\end{aligned}
$$

since $f_{1} d\left(\frac{f_{2}^{\prime}}{z}\right)-f_{2} d\left(\frac{f_{1}^{\prime}}{z}\right)=d\left(\frac{f_{1} f_{2}^{\prime}-f_{2} f_{1}^{\prime}}{z}\right)$;

$$
\begin{aligned}
& \sigma_{s}^{*}\left(\alpha_{3}\right)\left(f_{1} \partial+g_{1}, f_{2} \partial+g_{2}\right)=\operatorname{Res}_{z=0}\left(\frac{s f_{1}}{z}+g_{1}\right) d\left(\frac{s f_{2}}{z}+g_{2}\right) \\
& =\operatorname{Res}_{z=0}\left(g_{1} d g_{2}+s \frac{f_{1}}{z} d g_{2}+s g_{1} d\left(\frac{f_{2}}{z}\right)+s^{2} \frac{f_{1}}{z} d\left(\frac{f_{2}}{z}\right)\right) \\
& =\alpha_{3}+\operatorname{Res}_{z=0} s\left(\frac{f_{1}}{z} d g_{2}-\frac{f_{2}}{z} d g_{1}\right)+s^{2} \operatorname{Res}_{z=0} \frac{f_{1}}{z} d\left(\frac{f_{2}}{z}\right) \sim \alpha_{3},
\end{aligned}
$$

since

$$
\begin{aligned}
\operatorname{Res}_{z=0} \frac{f_{1}}{z} d\left(\frac{f_{2}}{z}\right) & =\operatorname{Res}_{z=0} \frac{1}{2}\left(\frac{f_{1}}{z} d\left(\frac{f_{2}}{z}\right)-\frac{f_{2}}{z} d\left(\frac{f_{1}}{z}\right)\right) \\
& =\operatorname{Res}_{z=0} \frac{1}{2 z^{2}}\left(f_{1} d f_{2}-f_{2} d f_{1}\right) . \text { Q.E.D. }
\end{aligned}
$$

We can now prove the following

(2.17) Proposition. Let $s \in \mathbb{C}$. Let $t_{s}: \mathscr{D} \rightarrow \mathscr{D}$ be defined as above, then

$$
\left.\begin{array}{l}
t_{s}^{*}\left(\alpha_{1}\right)=\alpha_{1} \\
t_{s}^{*}\left(\alpha_{2}\right)=\alpha_{2}+2 \alpha_{1} \\
t_{s}^{*}\left(\alpha_{3}\right)=\alpha_{3}-\alpha_{2}-\alpha_{1}
\end{array}\right\} .
$$


Proof. Using the above lemma we can assume $s=0$; let $t_{0}=t$, then it is clear that $t^{*}\left(\alpha_{1}\right)=\alpha_{1}$. Let us compute:

$$
\begin{aligned}
t^{*}\left(\alpha_{2}\right)\left(f_{1} \partial+g_{1}, f_{2} \partial+g_{2}\right) & =\operatorname{Res}_{z=0}\left[f_{1} d\left(f_{2}^{\prime \prime}+g_{2}^{\prime}\right)-f_{2} d\left(f_{1}^{\prime \prime}+g_{2}^{\prime}\right)\right] \\
& =\alpha_{2}+\operatorname{Res}_{z=0}\left(f_{1} d f_{2}^{\prime \prime}-f_{2} d f_{1}^{\prime \prime}\right)=\alpha_{2}+2 \alpha_{1}, \\
t^{*}\left(\alpha_{3}\right)\left(f_{1} \partial+g_{1}, f_{2} \partial+g_{2}\right) & =\operatorname{Res}_{z=0}\left[\left(f_{1}^{\prime}+g_{1}\right) d\left(f_{2}^{\prime}+g_{2}\right)\right] \\
& =\alpha_{3}+\operatorname{Res}_{z=0} f_{1}^{\prime} d f_{2}^{\prime}+\operatorname{Res}_{z=0}\left(f_{1}^{\prime} d g_{2}+g_{1} d f_{2}^{\prime}\right) \\
& =\alpha_{3}-\operatorname{Res}_{z=0} f_{1} d f_{2}^{\prime \prime}-\operatorname{Res}_{z=0}\left(f_{1} d g_{2}^{\prime}-f_{2} d g_{2}^{\prime}\right) \\
& =\alpha_{3}-\alpha_{2}-\alpha_{1} . \quad \text { Q.E.D. }
\end{aligned}
$$

It will be convenient for what follows to introduce a new basis for $H^{2}(\mathscr{D})$ and write $t^{*}$ with respect to this basis. Note that $\mathscr{D}$ acts naturally on $H$ by

$$
\phi_{0}(f \partial+g) h=f h^{\prime}+g h .
$$

So we get a representation

$$
\phi_{0}: \mathscr{D} \rightarrow \mathbf{a}_{\infty} .
$$

In the preceding section we defined a canonical 2-cocycle $\psi$ for $\mathbf{a}_{\infty}$. Let $\psi_{0}=\phi_{0}^{*}(-\psi)$. It is an easy computation to see that

$$
\psi_{0}=-\frac{\alpha_{1}}{6}+\frac{\alpha_{2}}{2}+\alpha_{3} .
$$

We now take as a new basis for $H^{2}(\mathscr{D})$ the set $\left\{\gamma, \psi_{0}, t^{*} \psi_{0}\right\}$, where $\gamma=-\frac{1}{6} \alpha_{1}$. It is then immediate to verify the following

(2.20) Corollary. With respect to the basis $\left\{\gamma, \psi_{0}, t^{*} \psi_{0}\right\}$ the homomorphism $t^{*}: H^{2}(\mathscr{D}) \rightarrow H^{2}(\mathscr{D})$ is represented by the matrix:

$$
t^{*}=\left(\begin{array}{rrr}
1 & 0 & 12 \\
0 & 0 & -1 \\
0 & 1 & 2
\end{array}\right) .
$$

In the sequel we shall also use the following basis of $H^{2}(\mathscr{D})$ :

$$
\left\{\psi_{0}, t^{*} \psi_{0}, t^{* 2} \psi_{0}\right\}
$$

Finally, notice that the Lie algebra $\mathbf{d}$ acts, by Lie bracket, on the ring of pseudodifferential operators

$$
\operatorname{Psd}=\left\{\sum_{i=-\infty}^{N} f_{i} \partial^{i}, f_{i} \in H\right\}
$$

and that it preserves the canonical filtration of Psd given by

$$
\operatorname{Psd}_{n}=\left\{\sum_{i=-\infty}^{-n} f_{i} \partial^{i}, f_{i} \in H\right\} \text {. }
$$


We then get an action of $d$ on the span of differentials of order $n$ :

$$
\Omega^{n}=H d z^{n}=H \partial^{-n}=\operatorname{Psd}_{n} / \operatorname{Psd}_{n+1},
$$

and hence a representation

$$
\varrho_{n}: \mathbf{d} \rightarrow \mathbf{a}_{\infty} .
$$

It is not hard to verify that $\varrho_{n}=\phi_{0} \circ t^{n} \circ i$, where $i: \mathbf{d} \rightarrow \mathscr{D}$ is the inclusion. A straightforward computation gives

$$
\varrho_{n}^{*}(\psi)=\left(6 n^{2}-6 n+1\right) \varrho_{1}^{*}(\psi)=\varrho_{1-n}^{*}(\psi) .
$$

\section{The Basic Varieties and the Krichever Construction}

We are now going to construct two infinite dimensional varieties $\hat{\mathscr{M}}_{g}$ and $\widehat{\mathscr{F}}_{g-1}$. The first one will parametrize triples $(C, p, z)$, where $C$ is a smooth curve of genus $g$, $p$ a point on $C$, and $z$ a local coordinate defined near $p$ and vanishing at $p$. The second one will parametrize quintuples $(C, p, z, L,[\phi])$, where $(C, p, z)$ are as above, $L$ is a degree $g-1$ line bundle on $C$, and $[\phi]$ is an equivalence class of local trivializations of $L$ near $p$, differing from each other by a non-zero multiplicative constant.

Let us start with a definition. Consider a family

$$
\mathscr{E} \underset{\sigma}{\stackrel{\pi}{\rightleftarrows}} T
$$

of pointed curves of genus $g$ parametrized by $T$. We say that $\mathscr{E}$ is a family of pointed curves with local parameters if there exists a neighbourhood $\mathscr{U}$ of the section $\sigma(T)$ and a holomorphic function $Z$ on $\mathscr{U}$, vanishing on $\sigma(T)$, such that for every $t$ in $T$ the function $z_{t}=\left.Z\right|_{U \cap \mathscr{E}_{t}}$ is a local coordinate around the point $\sigma(t)$ on the Riemann surface $\mathscr{E}_{t}$. The notion of isomorphism between families of pointed curves with local parameter is the obvious one. Notice also that given a smooth curve $C$ a point $p$ on $C$ and a local parameter $z$ around $p$, the triple $(C, p, z)$ admits only the trivial automorphism. Therefore, we may define a deformation of the triple $(C, p, z)$, simply as a family of pointed curves with local parameters $(\mathscr{E}, \pi, T, \sigma, Z)$ together with a point $t_{0} \in T$ and an identification of the "central data"

$$
\left(\mathscr{D}_{t_{0}}, \sigma\left(t_{0}\right), z_{t_{0}}\right)=(C, p, z),
$$

an isomorphism between deformations being simply an isomorphism between families (of pointed curves with local parameters) which is compatible with the identification of the central data.

Let us now consider a family of pointed curves

$$
\mathscr{C} \underset{\sigma}{\stackrel{\pi}{\rightleftarrows}} S \text {. }
$$

Given a point $s \in S$ choose a small neighbourhood $V$ of $s$ over which $\mathscr{C}$ trivializes in the $C^{\infty}$ sense. Look at a tubular neighbourhood $\mathscr{U}$ of the section $\left.\sigma(V) \subset \mathscr{C}\right|_{V}$, and think of it as a family discs parametrized by $V$. As such it must be holomorphically 
trivial. Let

$$
Z: \mathscr{U} \rightarrow \Delta \times V, \quad \Delta=\{z \in \mathbb{C}:|z|<\varepsilon\}
$$

be a trivialization of $\mathscr{U}$ such that $Z(\sigma(v))=(0, v), v \in V$. For every $v \in V$ the pointed curve $\left(\mathscr{C}_{v}, \sigma(v)\right)$ is then equipped with the local coordinate

$$
z_{v}=\left.Z\right|_{\mathscr{C}_{v} \cap U} .
$$

Consider the vector space

$$
H_{+}^{\prime}=\left\{h \in H_{+}: h(0)=0, h^{\prime}(0) \neq 0\right\}
$$

and consider the space $V \times H^{\prime}{ }_{+}$with its natural structure of infinite dimensional variety. Suppose now that $V^{\prime}$ is another neighbourhood of $s$ over which $\mathscr{C}$ trivializes, let $\mathscr{U}^{\prime}$ be a tubular neighbourhood of $\sigma\left(V^{\prime}\right)$ and $Z^{\prime}: \mathscr{U}^{\prime} \rightarrow \Delta \times V^{\prime}$ a holomorphic trivialization of $\mathscr{U}^{\prime}$, vanishing on $\sigma\left(V^{\prime}\right)$. Let $\Delta^{\prime} \subset \Delta$ be a disc such that $Z^{-1}\left(\Delta^{\prime} \times\left(V^{\prime} \cap V\right)\right) \subset \mathscr{U} \cap \mathscr{U}^{\prime}$. Consider

$$
\begin{aligned}
Z^{\prime} Z^{-1}: \Delta^{\prime} \times\left(V^{\prime} \cap V\right) & \rightarrow \Delta \times\left(V^{\prime} \cap V\right), \\
(z, v) & \mapsto(h(z, v), v) ;
\end{aligned}
$$

we then get an analytic map

$$
\begin{gathered}
\left(V^{\prime} \cap V\right) \times H_{+}^{\prime} \rightarrow\left(V^{\prime} \cap V\right) \times H_{+}^{\prime}, \\
(v, \zeta(z)) \mapsto(v, h(\zeta(z), v)) .
\end{gathered}
$$

Via this map we glue $V \times H_{+}^{\prime}$ to $V^{\prime} \cap H_{+}^{\prime}$ along $\left(V \cap V^{\prime}\right) \times H_{+}^{\prime}$. In this way we construct an infinite dimensional variety $\hat{S}$ whose points may be interpreted as pairs $\left(s, z_{s}\right)$, where $s \in S$ and $z_{s}$ is a local coordinate on $C_{s}$ near $\sigma(s)$. The variety $\hat{S}$ is equipped with a natural projection

$$
f: \widehat{S} \rightarrow S,
$$

and the fibers of $f$ are isomorphic to $H_{+}^{\prime}$. Pulling back $\mathscr{C}$ to $\hat{S}$ yields a family $(\hat{\mathscr{C}}, \hat{\pi}, \hat{S}, \hat{\sigma}, Z)$ of pointed curves with local parameters, parametrized by $\hat{S}$.

Using now Kuranishi families of pointed curves as building blocks [ACGH] we can construct by an obvious patching process a smooth infinite dimensional complex manifold $\hat{\mathscr{M}}_{g}$ modelled on $H_{+} \times \mathbb{C}^{3 g-1}$, whose points represent all the triples of the form $(C, p, z)$. Such a moduli space is equipped with a universal family of pointed curves with local parameters

$$
\hat{\mathscr{C}} \underset{\hat{\sigma}}{\stackrel{\hat{\pi}}{\rightleftarrows}} \hat{\mathscr{M}}_{g} .
$$

We also have a natural projection

$$
\begin{gathered}
\hat{\mathscr{M}}_{g} \stackrel{p^{\prime \prime}}{\rightarrow} \mathscr{M}_{g}^{\prime \prime} \\
(C, p, z) \mapsto\left(C, p, \frac{\partial}{\partial z}\right)
\end{gathered}
$$

and the fibers of $p^{\prime \prime}$ are isomorphic to $z+z^{2} H_{+}$(i.e. to $H_{+}$) so that, in particular, $p^{\prime \prime}$ induces an isomorphism

$$
H^{*}\left(\hat{\mathscr{M}}_{g}\right) \cong H^{*}\left(\mathscr{M}_{g}^{\prime \prime}\right)
$$


We leave it to the reader to verify that $\hat{\mathscr{M}}_{g}$ satisfies the obvious local (respectively global) universal properties with respect to deformations (respectively families) of pointed curves with local parameters.

Let us now introduce line bundles in our picture. Consider a pointed curve $(C, p)$. Let $L$ be a line bundle on $C$, and $\mathcal{O}(L)$ the corresponding $\mathcal{O}_{C}$-module. By a local trivialization $\phi$ of $L$ at $p$ we mean an isomorphism of $\mathscr{O}_{C}(L)_{C, p}$ with $\mathscr{O}_{C, p}$. Given two line bundles $L_{1}$ and $L_{2}$ with local trivializations $\phi_{1}$ and $\phi_{2}$ at $p$, we say that the pairs $\left(L_{1}, \phi_{1}\right)$ and $\left(L_{2}, \phi_{2}\right)$ are equivalent if there exists an isomorphism $\alpha: L_{1} \rightarrow L_{2}$ such that the homomorphism

$$
\phi_{2} \alpha \phi_{1}^{-1}: \mathcal{O}_{C, p} \rightarrow \mathcal{O}_{C, p}
$$

is the identity. We shall denote by $[L, \phi]$ the equivalence class of $(L, \phi)$. Clearly, the set of equivalence classes $[L, \phi]$ form a group under multiplication

$$
\left[L_{1}, \phi_{1}\right]\left[L_{2}, \phi_{2}\right]=\left[L_{1} \otimes L_{2}, \phi_{1} \otimes \phi_{2}\right]
$$

with the identity given by $\left[\mathcal{O}_{C}, 1\right]$. We denote this group by $M(C)$. We denote by $M^{0}(C)$ the subgroup of $M(C)$ consisting of pairs $[L, \phi]$ with $\operatorname{deg} L=0$, and by $M^{h}(C)$ the subset of $M(C)$ of pairs $[L, \phi]$ with $\operatorname{deg} L=h$. Clearly, forgetting about the trivialization yields the following commutative diagram of homomorphisms

$$
\begin{gathered}
M(C) \stackrel{\delta}{\longrightarrow} \operatorname{Pic}(C) \\
\uparrow \\
M^{0}(C) \longrightarrow \operatorname{Pic}^{0}(C),
\end{gathered}
$$

where the horizontal maps are surjective. Since $C$ is complete, so that the only global holomorphic functions on $C$ are constant, we immediately get that the kernel of $\delta$ may be identified with the subgroup of invertible elements in $\mathcal{O}_{C, p}$ whose value at $p$ is 1 . Therefore, upon choosing a local parameter $z$ on $C$ at $p$ vanishing at $p$, we can identify $\mathcal{O}_{C, p}$ with $H_{+}=\mathbb{C}\{z\}$ and $\operatorname{Ker} \delta$ with the multiplicative group in $H_{+}$of elements of the form $1+z f(z), f(z) \in H_{+}$, and thus with $H_{+}$itself. Starting from the triple $(C, p, z)$ we want to consider $M^{0}(C)$ as a quotient of $H$. To do this we define a surjective homomorphism

$$
\mu^{0}(C, p, z): H \rightarrow M^{0}(C)
$$

as follows. Given $f \in H$, let $\mathscr{U} \ni 0$ be a small enough neighbourhood such that $z$ and $f$ are defined on $\mathscr{U}$. Identify $\mathscr{U}$ with a neighbourhood of $p$ in $C$ via $z$. Let

$$
L=((C-p) \times \mathbb{C}) \amalg(\mathscr{U} \times \mathbb{C}) / \sim,
$$

where, given $(q, \alpha) \in(C-p) \times \mathbb{C},\left(q^{\prime}, \alpha^{\prime}\right) \in \mathscr{U} \times \mathbb{C}$,

$$
(q, \alpha) \sim\left(q^{\prime}, \alpha^{\prime}\right) \Leftrightarrow q=q^{\prime}, \quad \alpha^{\prime}=\alpha \exp f .
$$

By definition $L$ comes equipped with a canonical local trivialization which we denote by $\phi$. We set

$$
\mu^{0}(C, p, z)(f)=[L, \phi] .
$$

It is immediate to verify that $\mu^{0}$ is a surjective homomorphism whose kernel equals $\mathcal{O}(C-p)$ which we may consider as a subspace of $H$ using our trivialization. 
Furthermore, notice that we get a canonical bijection

$$
\gamma_{h}: M^{0}(C) \rightarrow M^{h}(C), \text { for any } h \in \mathbb{Z}
$$

given by $\gamma_{h}([L, \phi])=\left[L \otimes \mathcal{O}(h p), \phi z^{-h}\right]$. So we get a surjective map

Let now

$$
\mu^{h}(C, p, z): H \rightarrow M^{h}(C) \text {. }
$$

$$
\mathscr{C} \underset{\sigma}{\stackrel{\pi}{\rightleftarrows}} S
$$

be a family of pointed curves with local parameters.

Set

$$
\hat{\mathscr{F}}_{h}(\pi)=S \times H / \sim
$$

where

$$
(s, f) \sim\left(s^{\prime}, f^{\prime}\right) \Leftrightarrow s=s^{\prime}, \quad \mu^{h}\left(C_{s}, \sigma(s), z_{s}\right)(f)=\mu^{h}\left(C_{s}, \sigma(s), z_{s}\right)\left(f^{\prime}\right) .
$$

$\hat{\mathscr{F}}_{h}(\pi)$ is an infinite dimensional complex analytic space whose points represent pairs $(s,[L, \phi])$, where $s \in S$ and $[L, \phi] \in M^{h}\left(C_{s}\right)$. On $\hat{\mathscr{F}}_{h}(\pi)$ natural projections are defined:

$$
\begin{aligned}
& f: \hat{\mathscr{F}}_{h}(\pi) \rightarrow S, \\
& q: \hat{\mathscr{F}}_{h}(\pi) \rightarrow \operatorname{Pic}^{h}(\pi),
\end{aligned}
$$

where $\operatorname{Pic}^{h}(\pi)$ is the relative Picard variety of $\pi$. The fiber of $f$ over $s \in S$ is isomorphic to $M^{0}\left(C_{s}\right)$ while the fiber of $q$ over $(s, L) \in \operatorname{Pic}^{h}(\pi)$ is isomorphic to $H_{+}$. In particular,

$$
H^{*}\left(\mathscr{F}_{h}(\pi)\right)=H^{*}\left(\operatorname{Pic}^{h}(\pi)\right) .
$$

Consider now the universal family (3.2)

and set

$$
\hat{\mathscr{C}} \underset{\hat{\sigma}}{\stackrel{\hat{\pi}}{\rightleftarrows}} \hat{M}_{g},
$$

$$
\hat{\mathscr{F}}_{g-1}=\hat{\mathscr{F}}_{g-1}(\hat{\pi}) \text {. }
$$

From (3.8) and (3.3) it follows that [cf. (1.13)]

$$
H^{*}\left(\hat{\mathscr{F}}_{h}\right) \cong H^{*}\left(\operatorname{Pic}^{h}\left(\pi^{\prime \prime}\right)\right) .
$$

Given a smooth curve $C$, a point $p \in C$, a line bundle $L$ on $C$, and a local trivialization $\phi$ of $L$ at $p$, we denote by $[\phi]$ the set of all trivialization of $L$ at $p$ which differ from $\phi$ by a non-zero (multiplicative) constant. It is then clear that points of $\hat{\mathscr{F}}_{g-1}$ represent quintuples $(C, p, z, L,[\phi])$ modulo isomorphisms. We leave it to the reader to define the notion of family (respectively deformation) of quintuples $(C, p, z, L,[\phi])$, and verify that $\hat{\mathscr{F}}_{g-1}$ satisfies the obvious global (local) universal property.

Pick a point $x=(C, p, z, L,[\phi])$ in $\hat{\mathscr{F}}_{g-1}$. Consider the space of sections of $L$ on the open set $C-p$. A trivialization $\phi_{0} \in[\phi]$ and the local parameter $z$ make it possible to identify $\Gamma(C-p, L)$ with a linear subspace of $H$. Set

$$
W(x)=\Gamma(C-p, L) \subset H .
$$


Following the argument of [SW], one checks ${ }^{1}$ that $W(x)$ is a point in the infinite dimensional Grassmannian $\operatorname{Gr}(H)$ defined in Sect. 2 and that the resulting analytic map

$$
\begin{aligned}
W: \hat{\mathscr{F}}_{g-1} & \rightarrow \mathrm{Gr}(H) \\
x & \mapsto W(x)
\end{aligned}
$$

is injective. We call $W$ the Krichever map.

Consider now the diagram [cf. (1.6)]

$$
\begin{aligned}
& \hat{\mathscr{F}}_{g-1} \overbrace{}^{\tau=\tau_{2 g}-2} \\
& p \prod_{\xi}^{\hat{\mathscr{C}}} \stackrel{\hat{\pi}}{\longrightarrow} \hat{\mathscr{M}}_{g}
\end{aligned}
$$

where

$$
\left.\begin{array}{c}
\xi((C, p, z))=\left(C, p, z, \mathcal{O}_{c}((g-1) p),\left[z^{-g+1}\right]\right) \\
\tau((C, p, z, L,[\phi]))=\left(C, p, z, L \otimes \omega_{c}((2-2 g) p),\left[\phi z^{2 g-2} d z\right]\right)
\end{array}\right\},
$$

and define, by composition, the injective analytic maps

$$
W_{n}=W \tau^{n} \xi: \hat{\mathscr{M}}_{g} \rightarrow \operatorname{Gr}(H), \quad n \in \mathbb{Z} .
$$

Recall now the definition of the determinant line bundle $\mathscr{L}$ on $\operatorname{Gr}(H)$ (Sect. 2). From that definition and the exact sequence

$$
0 \rightarrow \Gamma(C, L) \rightarrow W(x) \rightarrow H_{-} \rightarrow H^{1}(C, L) \rightarrow 0
$$

it follows that

$$
W^{*} \mathscr{L}=\theta_{\hat{\pi}}^{-1} .
$$

Using now Lemma (1.7) and the fact that the relative dualizing sheaf $\omega$ is trivial on $\mathscr{M}_{g}^{\prime \prime}$, and hence on $\hat{\mathscr{M}}_{g}$, we get

$$
W_{n}^{*} \mathscr{L} \cong \lambda_{n}, \quad n \in \mathbb{Z}
$$

We are now going to study the diagram

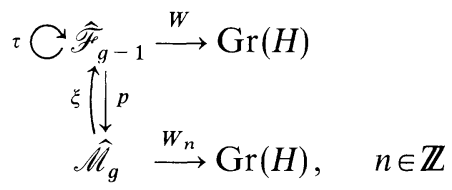

from the infinitesimal point of view, relating this study to that of the algebras $\mathscr{D}$ and $\mathbf{d}$ introduced in Sect. 2.

The first step in this analysis consists in describing the tangent bundles to $\hat{\mathscr{M}}_{g}$ and $\widehat{\mathscr{F}}_{g-1}$. To do this we are going to imitate the classical Kodaira-Spencer construction as described, for instance in [ACGH]. Namely, given a local universal family of curves

$$
\pi: \mathscr{C} \rightarrow \mathscr{M},
$$

${ }^{1}$ The fact that $p_{-}(W(x))$ has index 0 follows from the Riemann-Roch theorem. This is a reason why we use degree $g-1$ in the definition of $\hat{\mathscr{F}}_{g-1}$ 
the relative Picard variety

$$
p: \operatorname{Pic}^{g-1}(\pi) \rightarrow \mathscr{M},
$$

a point $[C] \in \mathscr{M}$ and a point $(C, L) \in \operatorname{Pic}^{g-1}(\pi)$ over $[C]$, the tangent space exact sequence

$$
0 \rightarrow T_{L}\left(\mathrm{Pic}^{g-1}(C)\right) \rightarrow T_{(C, L)}\left(\mathrm{Pic}^{g-1}(\pi)\right) \rightarrow T_{[C]}(\mathscr{M}) \rightarrow 0
$$

can be identified with the cohomology sequence,

$$
0 \rightarrow H^{1}\left(\mathcal{O}_{C}\right) \rightarrow H^{1}\left(\Sigma_{L}\right) \rightarrow H^{1}\left(\mathscr{T}_{C}\right) \rightarrow 0,
$$

where $\mathscr{T}_{C}$ is the tangent sheaf to $C$, and $\Sigma_{L}$ is the sheaf of differential operators of order less than or equal to 1 acting on sections of $L$.

To carry out this analysis in the infinite dimensional case we consider the Lie algebras $\mathbf{d}$ and $\mathscr{D}$ and points

$$
\begin{gathered}
x=(C, p, z, L,[\phi]) \in \widehat{\mathscr{F}}_{g-1}, \\
y=p(x)=(C, p, z) \in \widehat{\mathscr{M}}_{g} .
\end{gathered}
$$

A local trivialization $\phi_{0} \in[\phi]$ and the local parameter $z$ give natural identifications

$$
\mathcal{O}_{p}\left(\Sigma_{L}\right)=H_{+} \oplus H_{+} \partial \subset \mathscr{D}, \quad \mathcal{O}_{p}\left(\mathscr{T}_{C}\right)=H_{+} \partial \subset \mathbf{d},
$$

and natural Lie algebra inclusions

$$
\Gamma\left(C-p, \Sigma_{L}\right) \hookrightarrow \mathscr{D}, \quad \Gamma\left(C-p, \mathscr{T}_{C}\right) \hookrightarrow \mathbf{d} .
$$

We set

$$
\mathscr{D}_{x}=\Gamma\left(C-p, \Sigma_{L}\right), \quad \mathbf{d}_{y}=\Gamma\left(C-p, \mathscr{T}_{C}\right) .
$$

With this notation we have the following

(3.19) Proposition. For every $x \in \hat{\mathscr{F}}_{g-1}$, there is a commutative diagram

$$
\begin{aligned}
& 0 \longrightarrow \mathscr{D}_{x} \longrightarrow \mathscr{D}^{P_{\mathscr{D}}} T_{x}\left(\hat{\mathscr{F}}_{g-1}\right) \longrightarrow 0 \\
& \sigma\left\|\uparrow_{i} \quad \sigma\right\|\left\|_{i} \quad d p \downarrow \mid\right\| \xi \\
& 0 \longrightarrow \mathbf{d}_{y} \longrightarrow \mathbf{d} \stackrel{P_{\mathscr{O}}}{\longrightarrow} T_{y}\left(\hat{\mathscr{M}}_{g}\right) \longrightarrow 0
\end{aligned}
$$

such that the horizontal sequences are exact, where $\sigma$ is the symbol map and $i$ the natural inclusion. Moreover, there is a commutative diagram

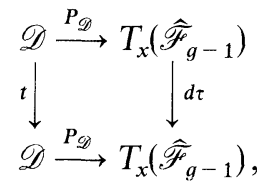

where $t=t_{2 g-2}$ and $\tau=\tau_{2 g-2}$ are as in (1.5) and (3.14).

Proof. By the universal property of $\hat{\mathscr{F}}_{g-1}$ we may identify the tangent space $T_{x}\left(\widehat{\mathscr{F}}_{g-1}\right)$ with the isomorphism classes of deformations of $(C, p, z, L,[\phi])$ parametrized by Spec $\mathbb{C}[\varepsilon] /\left(\varepsilon^{2}\right)=S$. Let $a(z)+b(z) \frac{\partial}{\partial z} \in \mathscr{D}$. Let $U_{0}$ be a small disc around 
$p$, where $z$ and $\phi$ are defined and such that $a(z)$ and $b(z)$ are holomorphic in $U_{0}-\{p\}$. Set $U_{1}=C-p$. Define a family of pointed curves with local parameter

by setting

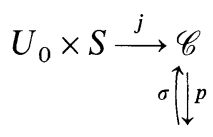

$S$

$$
\begin{gathered}
\mathscr{C}=\left(U_{0} \times S\right) \amalg\left(U_{1} \times S\right) / \sim, \\
(z, \varepsilon) \sim(z+\varepsilon b(z), \varepsilon), \quad z \in U_{0} \cap U_{1},
\end{gathered}
$$

and $p(z, \varepsilon)=\varepsilon$, and where $j$ is the natural inclusion. Define then a line bundle $\mathscr{L}$ and $\mathscr{C}$ by setting

$$
\begin{gathered}
\mathscr{L}=\left(\left.L\right|_{U_{0}} \times S\right) \amalg\left(\left.L\right|_{U_{1}} \times S\right) / \sim, \\
(\phi, \varepsilon) \sim(\phi(1+\varepsilon a(z)), \varepsilon), \quad z \in U_{0} \cap U_{1} .
\end{gathered}
$$

This line bundle is equipped with the obvious trivialization $(\phi, \varepsilon)$. The pair $(\mathscr{C}, \mathscr{L})$ is the deformation of $(C, p, z, L,[\phi])$ which we associate to $a(z)+b(z) \frac{\partial}{\partial z}$. This defines a homomorphism $P_{\mathscr{D}}$ from $\mathscr{D}$ to $T_{x}\left(\hat{\mathscr{F}}_{g-1}\right)$. It is a trivial matter to check that this homomorphism is surjective; let us check that its kernel is $\mathscr{D}_{x}$. Suppose first that $a(z)+b(z) \frac{\partial}{\partial z}$ is in the kernel. This means, in particular, that $(\mathscr{C}, \mathscr{L})$ as a deformation of $(C, L)$ is trivial. Thus by the Kodaira-Spencer theory $a(z)+b(z) \frac{\partial}{\partial z}$ lies in the image of the difference map

$$
\mathscr{D}_{x} \oplus \mathcal{O}_{p}\left(\Sigma_{L}\right) \stackrel{\mu}{\longrightarrow} \mathscr{D}
$$

[in fact, by Mayer-Vietoris, Coker $\left.\mu \cong H^{1}\left(\Sigma_{L}\right)\right]$. But it also means, by looking at the local parameter and at the local trivialization, that the isomorphism between $(\mathscr{C}, \mathscr{L})$ and the trivial deformation must induce the identity on $U_{0} \times S$ and (a nonzero constant multiple of) the identity on $\left.L\right|_{U_{0}} \times S$. This says that indeed $a(z)$ $+b(z) \frac{\partial}{\partial z}$ lies in $\mathscr{D}_{x}$. On the other hand, it is easy to check that if $a(z)+b(z) \frac{\partial}{\partial z}$ lies in $\mathscr{D}_{x}$ the corresponding family is isomorphic to a trivial deformation of $(C, p, z, L,[\phi])$. The statements about the tangent space to $\hat{\mathscr{M}}_{g}$ and the commutativity of the diagrams (3.20) and (3.21) follow in a completely analogous way. Q.E.D.

Let us now consider the map

$$
W: \widehat{\mathscr{F}}_{g-1} \rightarrow \operatorname{Gr}(H),
$$

and let $x=(C, p, z, L,[\phi])$ be a point in $\hat{\mathscr{F}}_{g-1}$; consider

$$
d W: T_{x}\left(\hat{\mathscr{F}}_{g-1}\right)=\mathscr{D} / \mathscr{D}_{x} \rightarrow T_{W(x)}(\operatorname{Gr}(H))=M(W(x), H / W(x)) .
$$


Consider a tangent vector $v \in T_{x}\left(\hat{\mathscr{F}}_{g-1}\right)$, let $A=a(z)+b(z) \frac{\partial}{\partial z}$ be such that $P_{\mathscr{D}}(A)=v$. Recalling (3.22) and (3.23) we see that for $f(z) \in W(x)$ we have:

$$
\begin{aligned}
d h(v)(f(z)) & =\varepsilon-\text { coeff. of }(1+\varepsilon a(z)) f(z+\varepsilon b(z)) \bmod W(x) \\
& =a(z) f(z)+b(z) \frac{\partial f(z)}{\partial z} \bmod W(x) \\
& =A(f) \bmod W(x)
\end{aligned}
$$

We can therefore conclude with the following

(3.24) Proposition. There is a commutative diagram

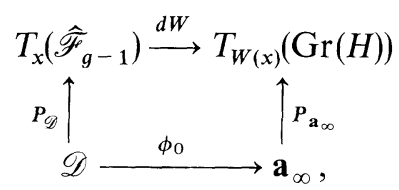

where $P_{\boldsymbol{a}_{\infty}}$ is the natural projection.

We summarize our infinitesimal analysis with the following commutative diagram of vector bundles:

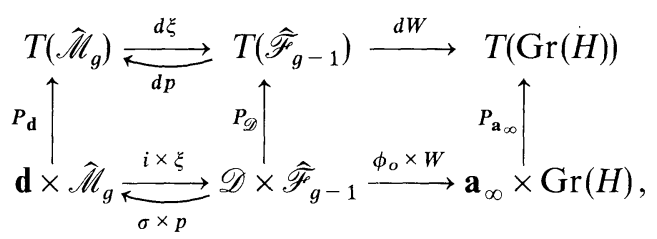

and we notice again that this diagram is compatible with the action of $t$ and $\mathscr{D}$ and $\tau$ on $\widehat{\mathscr{F}}_{g-1}$ :

$$
\begin{aligned}
& \mathscr{D} \times \hat{\mathscr{F}}_{g-1} \stackrel{P_{\mathscr{D}}}{\longrightarrow} T\left(\hat{\mathscr{F}}_{g-1}\right) \\
& t \times \tau \downarrow \\
& \downarrow \\
& \mathscr{D} \times \hat{\mathscr{F}}_{g-1} \stackrel{P_{\mathscr{D}}}{\longrightarrow} T\left(\hat{\mathscr{F}}_{g-1}\right) .
\end{aligned}
$$

We end this section by making a remark about the Krichever map linking the geometry of the Schubert varieties on the infinite dimensional Grassmannian with the gap sequences of Weierstrass points on Riemann surfaces. Given a line bundle $L$ on a Riemann surface $C$ the Weierstrass gap sequence of a point $p \in C$ with respect to $L$ is the sequence of integers $s_{1}, s_{2}, s_{3}, \ldots$ defined by

$$
\operatorname{dim} H^{0}\left(C, L\left(-s_{k} p\right)\right)=k \text {. }
$$

Recalling Lemma (1.18) we set

$$
\begin{aligned}
\Sigma_{S} & =\left\{W \in \operatorname{Gr}(H) \mid S \text { is the minimal index set for which } P_{S}: W \rightarrow H_{S} \text { is an iso }\right\} \\
& =\left\{W \in \operatorname{Gr}(H) \mid \lim _{\lambda \rightarrow 0} R_{\lambda} W=H_{S}\right\},
\end{aligned}
$$

where

$$
R_{\lambda} f(z)=f(\lambda z) .
$$

$\Sigma_{S}$ should be thought of as a Schubert cell and $H_{S}$ as its center. If $S=\left(s_{1}, s_{2}, \ldots\right)$, $s_{i}=-i, i \gg 0$, then

$$
\operatorname{codim} \Sigma_{S}=\sum_{i \geqq 1}\left(s_{i}+i\right) .
$$


Consider now the Krichever map

$$
W: \mathscr{F}_{g-1} \rightarrow \operatorname{Gr}(H),
$$

one can then observe that

$W^{-1}\left(\Sigma_{S}\right)=\{(C, p, z, L,[\varphi]): p$ has Weierstrass sequence $S$ with respect to $L\}$.

Essentially this remark goes back to Mumford (see $[\mathrm{Mu}]$, and a letter from Mumford to G. Segal) and could be of central importance in the understanding of the cohomology ring of $\mathscr{M}_{g}$.

\section{Line Bundles on the Basic Varieties}

In this section we shall relate the extensions of the Lie algebras $\mathbf{d}$ and $\mathscr{D}$ with the line bundles $\hat{\mathscr{M}}_{g}$ and $\hat{\mathscr{F}}_{g-1}$. We start with an easy result of a general nature.

(4.1) Lemma. Let $X$ be a (possibly infinite-dimensional) complex manifold. Denote by $\operatorname{Vect}(X)$ the Lie algebra of analytic vector fields on $X$, and let $\mathbf{g}$ be a subalgebra such that

i) $\forall x \in X$ the evaluation map $\phi_{x}: \mathbf{g} \rightarrow T_{x}(X)$ is surjective,

ii) $\mathbf{g}_{x}=\operatorname{Ker} \phi_{x}$ is such that $\left[\mathbf{g}_{x}, \mathbf{g}_{x}\right]=\mathbf{g}_{x}$.

Then for every Lie algebra continuous central extension

$$
0 \rightarrow \mathbb{C} \rightarrow \hat{\mathbf{g}} \rightarrow \mathbf{g} \rightarrow 0,
$$

which is trivial on $\mathbf{g}_{x}, \forall x \in X$, we can associate a continuous extension

$$
0 \rightarrow X \times \mathbb{C} \rightarrow E \rightarrow T(X) \rightarrow 0 .
$$

This defines a homomorphism

$$
\bigcap_{x \in X} \operatorname{Ker} r_{x} \rightarrow \operatorname{Ext}^{1}\left(\mathscr{T}_{X}, \mathcal{O}_{X}\right),
$$

where $r_{x}: H^{2}(\mathbf{g}, \mathbb{C}) \rightarrow H^{2}\left(\mathbf{g}_{x}, \mathbb{C}\right)$ is induced by the restriction, and where $\mathscr{T}_{X}$ denotes the tangent sheaf.

Proof. The evaluation maps and property i) give a surjective homomorphism

$$
X \times \mathbf{g} \rightarrow T(X),
$$

whose kernel is the vector bundle $V$ having $\mathbf{g}_{x}$ as fiber over $x$. Consider the diagram

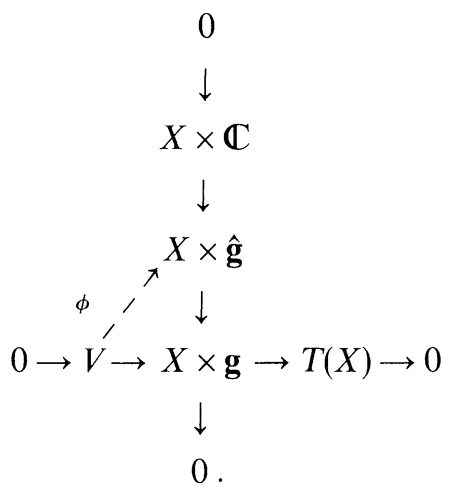


In order to define the homomorphism $\phi$ we need to find for every $x \in X$ a canonical splitting of the preimage $\hat{\mathbf{g}}_{x}$ of $\mathbf{g}_{x}$ in $\hat{\mathrm{g}}$. We know that for any $x \in X, \hat{\mathbf{g}}_{x}=\mathbf{h} \oplus \mathbb{C}$ as a Lie algebra in a possibly non-canonical way, where $\mathbf{h}$ is a closed subalgebra isomorphic to $\mathbf{g}_{x}$. On the other hand, $\mathbf{h}$ contains the commutator subalgebra $\left[\hat{\mathbf{g}}_{x}, \hat{\mathbf{g}}_{x}\right]$ as a dense subspace. Thus the above splitting is canonically defined. Q.E.D.

In order to apply this lemma to the case of Riemann surfaces we need the following result:

(4.3) Lemma. Let $C$ be an affine Riemann surface, and L a holomorphic line bundle on C. Denote by $x$ the pair $(C, L)$, set $\mathbf{d}_{x}=\operatorname{Vect}(C)$ and let $\mathscr{D}_{x}$ denote the algebra of global differential operators of order $\leqq 1$ acting on sections of $L$. Then: $\left[\mathbf{d}_{x}, \mathbf{d}_{x}\right]=d_{x}$, $\left[\mathscr{D}_{x}, \mathscr{D}_{x}\right]=\mathscr{D}_{x}$.

Proof. We shall prove the lemma only for $\mathscr{D}_{x}$, the proof for $\mathbf{d}_{x}$ being similar and easier. Since every line bundle on $C$ is analytically trivial we can assume that $L=C \times \mathbb{C}$, so that $\mathscr{D}_{x}$ is the algebra of global differential operators of order less or equal than one acting on functions. Choose a nowhere vanishing vector field $\partial$ on $C$, so that any element of $\mathscr{D}_{x}$ can be written uniquely as $f \partial+g, f, g \in \mathscr{O}_{C}$. We first notice that $\left[\mathscr{D}_{x}, \mathscr{D}_{x}\right]$ is an $\mathscr{O}_{C}$-module:

$$
s[f \partial+g, h \partial+k]=\left[s f \partial, \frac{1}{2} h \partial+k\right]+\left[\frac{1}{2} f \partial+g, s h \partial\right] .
$$

Then it suffices to show that the elements $\partial$ and 1 lie in $\left[\mathscr{D}_{x}, \mathscr{D}_{x}\right]$. In case $C$ is an open set in $\mathbb{A}^{1}$ and $\partial=\frac{\partial}{\partial z}$, this is clear since $\partial=[\partial, z \partial], 1=[\partial, z]$. In general choose two projections $p_{1}, p_{2}: C \rightarrow \mathbb{A}^{1}$ with disjoint ramification divisors. Then $\partial_{i}=p_{i}^{*}\left(\frac{\partial}{\partial z}\right), i=1,2$, are rational vector fields with poles along the ramification divisors, and no zeroes. Thus there exist regular functions $f_{i}, i=1,2$ such that $f_{i} \partial_{i}$ are regular vector fields having zeroes at most along the ramification divisors. Set then

$$
f_{i} \partial_{i}=a_{i} \partial, \quad p_{i}^{*}(z)=z_{i}, \quad i=1,2 .
$$

We then have $a_{i} \partial\left(z_{i}\right)=f_{i}$, hence

$$
\left[a_{i} \partial, z_{i}\right]=f_{i}, \quad a_{i}\left[\partial, z_{i} \partial\right]=f_{i} \partial, \quad i=1,2 .
$$

Since $f_{i}$ and $f_{2}$ are relatively prime the lemma is proved. Q.E.D.

Putting together the results of our previous section we get

(4.5) Theorem. There exist canonical homomorphisms

$$
\begin{aligned}
\mu: H^{2}(\mathscr{D}) & \rightarrow \operatorname{Ext}^{1}\left(\mathscr{T}_{\hat{\mathscr{F}}_{g-1}}, \mathcal{O}_{\hat{\mathscr{F}}_{g-1}}\right)=H^{1}\left(\Omega_{\mathscr{F}_{g-1}}^{1}\right), \\
v: H^{2}(\mathbf{d}) & \rightarrow \operatorname{Ext}^{1}\left(\mathscr{T}_{\hat{\mathscr{M}}_{g}}, \mathcal{O}_{\hat{\mathcal{M}}_{g}}\right)=H^{1}\left(\Omega_{\hat{\mathcal{M}}_{g}}^{1}\right) .
\end{aligned}
$$

Proof. In order to apply Lemma (4.1) and more precisely the homomorphism (4.2), we have to show that given any extension of $\mathscr{D}$ (respectively $\mathbf{d}$ ) its restriction to $\mathscr{D}_{x}$ (respectively $\mathbf{d}_{x}$ ) is trivial for every $x \in \hat{\mathscr{F}}_{g-1}$ (respectively $x \in \hat{\mathscr{M}}_{g}$ ). Now we have 
seen that a basis of $H^{2}(\mathscr{D})$ is given by $\psi_{0}, t^{*} \psi_{0}, t^{*} \psi_{0}$ [cf. (2.22)] and that these are all pullback of the extension $\psi$ of $\mathbf{a}_{\infty}$. Since $\mathscr{D}_{t x}=(d \tau) \mathscr{D}_{x}$, it then suffices to show that the extension $\psi$ is trivial on $\phi_{0}\left(\mathscr{D}_{x}\right)$ for every $x$ [recall (2.19)]. On the other hand, $\phi_{0}\left(\mathscr{D}_{x}\right)$ is contained in the Lie algebra $\mathbf{a}_{W}=\mathbf{a}_{W(x)} \subset \mathbf{a}_{\infty}$, which is the Lie algebra of the stabilizer of $W=W(x)$. Denote by $\tilde{\mathbf{a}}_{W}$ the preimage of $\mathbf{a}_{W}$ in $\tilde{\mathbf{a}}_{\infty} ;$ it then suffices to show that $\tilde{\mathbf{a}}_{W}$ is a trivial extension of $\mathbf{a}_{W}$. Now the adjoint action of $\tilde{A}_{\infty}$ on $\tilde{\mathbf{a}}_{\infty}$ factors through $A_{\infty}$ so that if $W=g H_{-}$, then $\operatorname{ad} g\left(\mathbf{a}_{H_{-}}\right)=\mathbf{a}_{W}$ and $\operatorname{ad} g\left(\tilde{\mathbf{a}}_{H_{-}}\right)=\tilde{\mathbf{a}}_{W}$. Since $\tilde{\mathbf{a}}_{H_{-}}$is a trivial extension of $\mathbf{a}_{H_{-}}$also $\tilde{\mathbf{a}}_{W}$ is a trivial extension of $\mathbf{a}_{W}$. Q.E.D.

(4.6) Lemma. $\left[\overline{\left.\mathbf{a}_{W}, \mathbf{a}_{W}\right]}=\mathbf{a}_{W}, \forall W \in \operatorname{Gr}(H)\right.$.

Proof. It suffices to show this for $\mathbf{a}_{H_{-}}$. Assume there is a continuous Lie algebra homomorphism

$$
\mu: \mathbf{a}_{H_{-}} \rightarrow \mathbb{C} .
$$

Restrict $\mu$ to the elements in $\mathbf{a}_{H_{-}}$of type

$$
\left(\begin{array}{ll}
a_{++} & 0 \\
0 & 0
\end{array}\right) \quad \text { with } \quad a_{++}=\left(\begin{array}{ll}
0 & 0 \\
0 & A
\end{array}\right), \quad A \text { finite. }
$$

Then by the finite dimensional result $\mu$ restricts to a multiple of the trace. By varying the size of $A$ we see that this multiple is independent of the size of $A$. On the other hand, a general element $a_{++}$has no trace (e.g. $a_{++}=I$ ). Reasoning in the same way for the lower right block we conclude that $\mu$ must vanish on the elements of the form

$$
\left(\begin{array}{ll}
a_{++} & 0 \\
0 & a_{--}
\end{array}\right) .
$$

Since the Lie algebra consisting of elements of the form

$$
\left(\begin{array}{ll}
0 & 0 \\
* & 0
\end{array}\right)
$$

is contained in $\overline{\left[\mathbf{a}_{H_{-}}, \mathbf{a}_{H_{-}}\right]}$the lemma follows. Q.E.D.

Applying the general Lemma (4.1) we can associate to the extension $\hat{\mathbf{a}}_{\infty}$ an extension $\Sigma$ of $\mathscr{T}_{\mathrm{Gr}(H)}$ by $\mathcal{O}_{\mathrm{Gr}(H)}$.

(4.7) Lemma. $\Sigma$ is the sheaf $\Sigma_{\mathscr{L}}$ of differential operators of order less then or equal to 1 acting on sections of the determinant bundle $\mathscr{L}$.

Proof. Consider

$$
0 \rightarrow \mathcal{O}_{\mathrm{Gr}} \rightarrow \Sigma \rightarrow \mathscr{T}_{\mathrm{Gr}} \rightarrow 0 .
$$

It is easy to see that the canonical action of $\tilde{\mathbf{a}}_{\infty}$ on $\mathscr{L}$ is given by differential operators of order less than or equal to 1 . From this, and from the fact that the global sections of $\Sigma$ in $\tilde{\mathbf{a}}_{\infty}$ generate $\Sigma$, we get a homomorphism of sheaves

$$
\Sigma \stackrel{\sigma}{\longrightarrow} \Sigma_{\mathscr{L}}
$$


such that the diagram

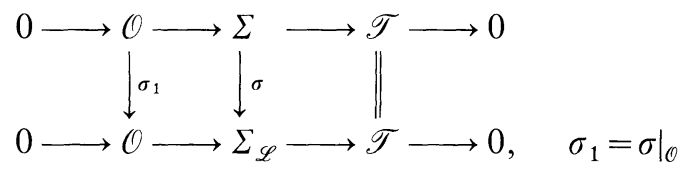

commutes. It is then easy to see that $\sigma_{1}$ is non-zero. Since the only global holomorphic functions on $\operatorname{Gr}(H)$ are constants, $\sigma_{1}$ must be an isomorphism. Thus $\sigma$ too is an isomorphism. Q.E.D.

Of course we have a canonical homomorphism

$$
c: H^{1}\left(\mathcal{O}_{\mathscr{F}_{\mathrm{g}-1}}^{*}\right) \longrightarrow \operatorname{Ext}^{1}\left(\mathscr{T}_{\hat{\mathscr{F}}_{\mathrm{g}-1}}, \mathcal{O}_{\hat{\mathscr{F}}_{\mathrm{g}-1}}\right)
$$

which associates to the isomorphism class of a line bundle $L$ on $\widehat{\mathscr{F}}_{g-1}$ the extension class given by the sheaf $\Sigma_{L}$ of differential operators of order less than or equal to 1 acting on $L$. Similarly we have

$$
c: H^{1}\left(\mathcal{O}_{\hat{M}_{g}}^{*}\right) \longrightarrow \operatorname{Ext}^{1}\left(\mathscr{T}_{\hat{\mathscr{M}}_{g}}, \mathcal{O}_{\hat{\mathscr{M}}_{g}}\right) .
$$

Notice that the homomorphism (4.8) commutes with the action of the automorphism $\tau: \widehat{\mathscr{F}}_{g-1} \rightarrow \widehat{\mathscr{F}}_{g-1}$.

(4.10) Theorem. Consider the canonical homomorphisms

$$
\begin{gathered}
\mu: H^{2}(\mathscr{D}) \longrightarrow \operatorname{Ext}^{1}\left(\mathscr{T}_{\hat{\mathscr{F}}_{g-1}}, \mathcal{O}_{\hat{\mathscr{F}}_{g-1}}\right)=H^{1}\left(\Omega_{\hat{\mathscr{F}}_{g-1}}^{1}\right), \\
v: H^{2}(\mathbf{d}) \longrightarrow \operatorname{Ext}^{1}\left(\mathscr{T}_{\hat{\mathscr{M}}_{g-1}}, \mathcal{O}_{\hat{\mathscr{M}}_{g}}\right)=H^{1}\left(\Omega_{\hat{\mathscr{M}}_{g}}^{1}\right) .
\end{gathered}
$$

Then, (cf. the definition of $\psi_{0}$ in Sect. 2)

i) $\mu\left(\psi_{0}\right)=c(\theta)$

ii) $\mu t^{*}=\tau^{*} \mu$,

iii) $\operatorname{Im} \mu=$ Linear span of $\operatorname{Im} c$,

iv) $v \varrho_{n}^{*}\left(\psi_{0}\right)=c\left(\lambda_{n}\right)$.

Proof. Consider the diagram (3.25)

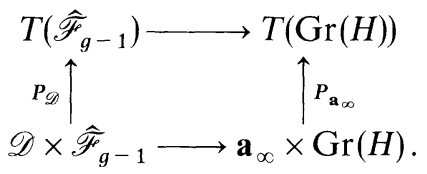

Recall that $w^{*}\left(\mathscr{L}^{-1}\right)=\theta$. Then, by functoriality, the pullback via $W$ of $\Sigma_{\mathscr{L}-1}$ is $\Sigma_{\theta}$. On the other hand $\phi_{0}^{*}(-\psi)=\psi_{0}$, so that by Lemma $(4.7) \mu\left(\psi_{0}\right)=c(\theta)$. The second statement follows immediately from the commutativity of diagram (3.26). Since $H^{2}(\mathscr{D})$ considered as a $\mathbb{Z}$-module via the action of $t^{*}$, is cyclic with generator $\psi_{0}$, iii) follows from i) and ii). Finally iv) follows from i), ii) and the commutativity of the diagram

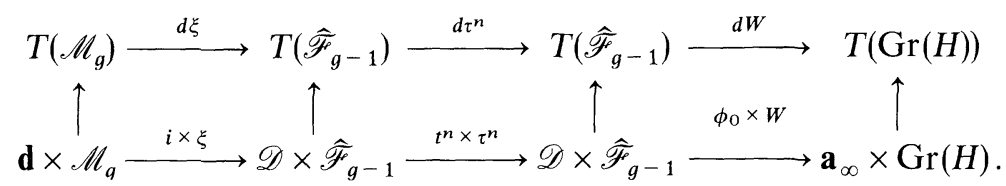

From iv) of the preceding theorem and (2.24) we get

Q.E.D. 
(4.11) Corollary. $c\left(\lambda_{n}\right)=\left(6 n^{2}-6 n+1\right) c\left(\lambda_{1}\right)$.

As is well known, using the Grothendieck-Riemann-Roch theorem, Mumford [M], shows that there is an equality of Chern classes

$$
c_{1}\left(\lambda_{n}\right)=\left(6 n^{2}-6 n+1\right) c_{1}\left(\lambda_{1}\right) .
$$

It is then natural to ask whether the preceding corollary implies this relation. This is indeed so. Consider first the diagram

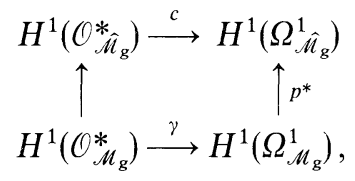

where the vertical arrows are induced by the natural projection and where, as usual, given a line bundle $L$, defined by transition functions $g_{\alpha \beta}$, we have $\gamma([L])$ $=d \log g_{\alpha \beta}$. Observe that $p^{*}$ is injective on $\operatorname{Im} \gamma$. In fact $p$ is obtained as composition

$$
\hat{\mathscr{M}}_{g} \stackrel{p_{1}}{\longrightarrow} \mathscr{M}_{g}^{\prime \prime} \stackrel{p_{2}}{\longrightarrow} \mathscr{M}_{g}^{(1)} \stackrel{p_{3}}{\longrightarrow} \mathscr{M}_{g}
$$

where $p_{3}^{*}$ is injective, $p_{2}^{*}\left(d \log g_{\alpha \beta}\right)=0$ if and only if $L$ is the dualizing sheaf $\omega$, and $p_{1}^{*}\left(d \log g_{\alpha \beta}\right)=0$ if and only if $L$ is trivial, being $\widehat{\mathscr{M}}_{g}$ an $H_{+}$-fibration over $\mathscr{M}_{g}^{\prime \prime}$. Now the Chern class

$$
c_{1}: H^{1}\left(\mathcal{O}^{*} \mathscr{M}_{g}\right) \rightarrow H^{2}\left(\mathscr{M}_{g}\right)
$$

is obtained by considering the de Rham class of $d \log g_{\alpha \beta}$. Thus we need to prove that $d \log g_{\alpha \beta}$ is $d$-exact if it is $\bar{\partial}$-exact. If $\mathscr{M}_{g}$ were complete we would have, by Hodge theory, a natural inclusion $H^{1}\left(\Omega_{\mathscr{M}_{\mathrm{g}}}^{1}\right) \rightarrow H^{2}\left(\mathscr{M}_{g}, \mathbb{C}\right.$ ) (in fact we believe that such an injection exists in our case). A possible way around this is given by Harer's theorem $[\mathrm{H}]$. By this theorem $L$ is a multiple of $\lambda_{1}$, and $\lambda_{1}$ is positive on $\mathscr{M}_{g}$. Suppose then that $d \log g_{\alpha \beta}$ is $\bar{\partial}$-exact, hence $d$-exact on any compact curve in $\mathscr{M}_{g}$. Integrating $d \log g_{\alpha \beta}$ over a compact curve contained in $\mathscr{M}_{g}$ we get zero, therefore implying that $L$ is trivial on $\mathscr{M}_{g}$ i.e. $d \log g_{\alpha \beta}$ is $d$-exact.

From our discussion we also get that, using Harer's theorem, there is a canonical isomorphism for $g \geqq 3$

$$
v: H^{2}(\mathbf{d}) \stackrel{\cong}{\longrightarrow} H^{2}\left(\mathscr{M}_{g}, \mathbb{C}\right) .
$$

Using the results of the next section, namely Corollary (5.7), we will also get a canonical isomorphism for $g \geqq 5$

$$
\mu: H^{2}(\mathscr{D}) \rightarrow H^{2}\left(\mathscr{F}_{g-1}^{\prime \prime}, \mathbb{C}\right),
$$

where $\mathscr{F}_{g-1}^{\prime \prime}$ is the relative Picard variety of

$$
\pi^{\prime \prime}: \mathscr{C}^{\prime \prime} \rightarrow \mathscr{M}_{g}^{\prime \prime} .
$$

\section{A Cohomology Computation}

Let $\mathscr{M}_{g}, g \geqq 5$, be the moduli space of curves of genus $g$. Let $\mathscr{M} \subset \mathscr{M}_{g}$ be the Zariski open set corresponding to automorphism free curves. Let $\Gamma_{g}$ be the mapping class 
group. It is well known $[\mathrm{H}]$ that

$$
H^{2}\left(\mathscr{M}_{g}, \mathbb{Z}\right) \cong H^{2}(\mathscr{M}, \mathbb{Z})=H^{2}\left(\Gamma_{g}\right) .
$$

Let

$$
\mathscr{M}^{(1)} \rightarrow \mathscr{M}
$$

be the universal family of curves over $\mathscr{M}$ and let $\mathscr{C} \stackrel{\pi}{\longrightarrow} \mathscr{M}^{(1)}$ be the universal family of pointed curves over $\mathscr{M}^{(1)}$. Consider the relative Picard variety

$$
\operatorname{Pic}^{h}(\pi) \rightleftarrows \mathscr{M}^{(1)}
$$

When $h=g-1$, we have as in (1.5) the translation homomorphism

$$
t: \mathscr{F}_{g-1} \rightarrow \mathscr{F}_{g-1} .
$$

We are going to prove the following

(5.3) Theorem. $H^{2}\left(\mathscr{F}_{h}, \mathbb{Z}\right) \cong \mathbb{Z}^{4}, H^{1}\left(\mathscr{F}_{h}, \mathbb{Z}\right)=0$.

Proof. In view of the isomorphism (1.4) we shall work with either $h=0$ or $h=g-1$. We first exhibit four linearly independent elements in $H^{2}\left(\mathscr{F}_{g-1}, \mathbb{Z}\right)$. To do this we consider, as in the first section, the line bundles $\theta, \theta_{t}, \lambda, \omega$ on $\widetilde{\mathscr{F}}_{g-1}$, where $\lambda$ is the pull-back, via $p$, of the Hodge bundle $\lambda_{1}$ on $\mathscr{M}^{(1)}$ and $\omega$ is the pull-back, via $p$, of $\omega_{\mathscr{M}^{(1)} / \mathscr{M}}$. With this notation the case $n=2$ of Corollary (1.12) together with the relation $\lambda_{2}=13 \lambda_{1}$ gives

$$
t^{*} \theta_{t}=\theta_{t}^{2} \otimes \theta^{-1} \otimes \lambda^{-12} \otimes \omega^{8\left(\frac{g}{2}\right)} .
$$

On the other hand one has

$$
t^{*} \lambda=\lambda, t^{*} \omega=\omega, t^{*} \theta=\theta_{t} \quad \text { (by definition). }
$$

Therefore, if we denote by $\Lambda$ the free abelian group $\mathbb{Z} \omega \oplus \mathbb{Z} \lambda \oplus \mathbb{Z} \theta \oplus \mathbb{Z} \theta_{t}$, the matrix of

$$
t^{*}: \Lambda \rightarrow \Lambda
$$

is

$$
\left(\begin{array}{cccc}
1 & 0 & 0 & 8\left(\begin{array}{l}
g \\
2
\end{array}\right) \\
0 & 1 & 0 & -12 \\
0 & 0 & 0 & -1 \\
0 & 0 & 1 & 2
\end{array}\right) .
$$

Now, it is easily seen that this matrix is nilpotent with Jordan partition $(3,1)$ and that

$$
\operatorname{Ker}\left(t^{*}-1\right)=\mathbb{Z} \lambda \oplus \mathbb{Z} \omega
$$

Consider now the map

$$
c: \Lambda \rightarrow H^{2}\left(\mathscr{F}_{g-1}, \mathbb{Z}\right)
$$

given by the Chern class. Since $t$ induces a map on $H^{2}\left(\mathscr{F}_{g-1}\right)$ which is compatible with $t^{*}$, the kernel of $c$ is $t^{*}$-stable, and hence contains a vector fixed by $t^{*}$. This 
vector must then lie in $\mathbb{Z} \lambda \oplus \mathbb{Z} \omega$. On the other hand the existence of the section $\xi$ in (5.2) shows that the homomorphism

$$
p^{*}: H^{2}\left(\mathscr{M}^{(1)}, \mathbb{Z}\right) \rightarrow H^{2}\left(\mathscr{F}_{g-1}, \mathbb{Z}\right)
$$

is an injection with $p^{*}\left(\left[\lambda_{1}\right]\right)=c(\lambda), p^{*}\left(\left[\omega_{\mathcal{M}^{(1)} / \mathcal{M}}\right]\right)=c(\omega)$, and, as well known [AC], $H^{2}\left(\mathscr{M}^{(1)}, \mathbb{Z}\right)$ is freely generated by $\left[\lambda_{1}\right]$ and $\left[\omega_{\mathscr{M}^{(1)} / \mathcal{M}}\right]$. It follows that $c$ is an injection. We now show that the number of generators of $H^{2}\left(\mathscr{F}_{h}, \mathbb{Z}\right)$ is not greater than 4 . Let $S$ be a topological compact oriented surface of genus $g$, and $\mathscr{T}_{g}=\mathscr{T}_{g}(S)$ the corresponding Teichmüller space. Set $\pi_{1}=\pi_{1}(S, q), H_{1}=H_{1}(S, \mathbb{Z})$. Consider the mapping class group $\Gamma_{g}^{1}$ of the pointed surface $(S, q)$ and the extension $\Gamma^{\prime}$ of $\Gamma$ by $H_{1}$, given by the natural action of $\Gamma$ on $H_{1}$. Finally consider the fiber product

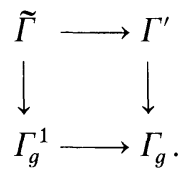

It is clear that $\tilde{\Gamma}$ is an extension of $\Gamma$ by $\pi_{1} \times H_{1}$ and that $\tilde{\Gamma}$ acts quasi-freely on $\mathscr{T}_{g} \times \Delta \times \mathbb{C}^{g}=X .(\Delta=\{z \in \mathbb{C}:|z|=1\})$. Removing the points of $X$ with nontrivial isotropy yields, by factoring the action of $\widetilde{\Gamma}$, an open set of $\mathscr{F}_{0}$ whose complement in $\mathscr{F}_{0}$ has high codimension. Therefore

$$
H^{i}\left(\widetilde{F}_{0}, \mathbb{Z}\right) \cong H^{i}(\tilde{\Gamma}, \mathbb{Z}), \quad i=1,2 .
$$

Consider the spectral sequence associated to the short exact sequence

and recall that by $[\mathrm{H}]$,

$$
1 \rightarrow \pi_{1} \times H_{1} \rightarrow \tilde{\Gamma} \rightarrow \Gamma \rightarrow 1,
$$

$$
H^{2}(\Gamma, \mathbb{Z}) \cong \mathbb{Z}, \quad H^{1}\left(\Gamma, H_{1}\right)=0, \quad H^{1}(\Gamma, \mathbb{Z})=0 .
$$

Also notice that

$$
H^{1}\left(\pi_{1}\right) \cong H_{1}, H^{2}\left(\pi_{1}\right) \cong \mathbb{Z}, H^{1}\left(H_{1}\right) \cong H_{1}, H^{2}\left(H_{1}\right) \cong \Lambda^{2} H_{1}
$$

By Kunneth formula we get

$$
H^{2}\left(\pi_{1} \times H_{1}\right) \cong \mathbb{Z} \oplus\left(H_{1} \otimes H_{1}\right) \oplus \Lambda^{2} H_{1}
$$

where the action of $\Gamma$ on $H_{1}$ factors through the homomorphism of $\Gamma$ into $\operatorname{Sp}(2 g, \mathbb{Z})$. Thus an easy computation of invariants yields

$$
\begin{gathered}
H^{0}\left(\Gamma, H^{1}\left(H_{1}\right)\right)=0, \\
H^{0}\left(\Gamma, H^{2}\left(\pi_{1} \times H_{1}\right)\right) \cong \mathbb{Z} \oplus \mathbb{Z} \oplus \mathbb{Z} .
\end{gathered}
$$

The $E^{2}$ term of the Hochschild-Serre spectral sequence is

$$
\begin{array}{rrrr}
\mathbb{Z}^{3} & \ldots & & \\
0 & 0 & \ldots & \\
\mathbb{Z} & 0 & \mathbb{Z} & \ldots
\end{array}
$$

This shows at the same time that $H^{1}(\tilde{\Gamma}, \mathbb{Z})=0$ and that $H^{2}(\tilde{\Gamma}, \mathbb{Z})$ has at most 4 generators. Q.E.D. 
Consider now the family of pointed curves with tangent vector (1.13)

$$
\mathscr{C}^{\prime \prime} \underset{\sigma^{\prime \prime}}{\stackrel{\pi^{\prime \prime}}{\rightleftarrows}} \mathscr{M}_{g}^{\prime \prime},
$$

and let

$$
\mathscr{F}_{h}^{\prime \prime}=\operatorname{Pic}^{h}\left(\pi^{\prime \prime}\right) .
$$

A simple spectral sequence argument based on Theorem (5.3) yields the following (5.7) Corollary. $H^{1}\left(\mathscr{F}_{h}^{\prime \prime}, \mathbb{Z}\right)=0, H^{2}\left(\mathscr{F}_{h}^{\prime \prime}, \mathbb{Z}\right) \cong \mathbb{Z}^{3}$. Moreover the elements $\lambda_{1}, \theta$. $n_{\text {, are }}$ linearly independent in $H^{2}\left(\mathscr{F}_{h}^{\prime \prime}, \mathbb{Z}\right)$ and the translation isomorphism $t^{*}$ of $\mathbb{Z} \lambda_{1} \oplus \mathbb{Z} \theta$ $\oplus \mathbb{Z} \theta_{t}$ into itself is given by the matrix

$$
\left(\begin{array}{ccc}
1 & 0 & -12 \\
0 & 0 & -1 \\
0 & 1 & 2
\end{array}\right) .
$$

(Notice that the above matrix does not depend on g.) Furthermore the natural projection induces identifications

$$
\mathbb{Z} \lambda_{1}=H^{2}\left(\mathscr{M}_{g}\right)=H^{2}\left(\mathscr{M}_{g}^{\prime \prime}\right)=H^{2}\left(\mathscr{F}_{g-1}^{\prime \prime}\right)^{t^{*}} .
$$

\section{Appendix: A Representation Theory of the Universal Central Extension of the Lie Algebra $\mathscr{D}^{F}$}

Let $\hat{\mathscr{D}}$ be the universal central extension of the Lie algebra $\mathscr{D}^{F}$. Due to Proposition 2.1 (3), the center of $\hat{\mathscr{D}}$ is 3-dimensional and we can choose a basis $d_{m}, z^{m}(m \in \mathbb{I}), c, c_{a}, c_{3}$ of $\hat{\mathscr{D}}$ such that $c, c_{a}, c_{3}$ is a basis of the center of $\hat{\mathscr{D}}$, and the following commutation relations hold:

$$
\begin{aligned}
& {\left[d_{m}, d_{n}\right]=(m-n) d_{m+n}+\frac{1}{12} \delta_{m,-n}\left(m^{3}-m\right) c,} \\
& {\left[z^{m}, z^{n}\right]=m \delta_{m,-n} c_{a},} \\
& {\left[z^{m}, d_{n}\right]=m z^{m+n}-\sqrt{-1} m^{2} \delta_{m,-n} c_{3} .}
\end{aligned}
$$

Note that in the quotient by the center, $d_{m}$ may be identified with $-z^{m+1} \frac{d}{d z}$. The choice of the cocycle in (6.3) is the most convenient one for the study of unitary representations of $\hat{\mathscr{D}}$. Note that $\hat{\mathscr{D}}$ is also the universal central extension of the semidirect product of Lie algebras (6.1) (the Virasoro algebra Vir) and (6.2) (the oscillator algebra $=$ affine algebra associated to the 1 -dimensional Lie algebra) by a 1-dimensional center, in a sharp contrast with the non-abelian case [in which (6.2) is replaced by an affine algebra associated to a simple Lie algebra], when there is no further non-trivial central extension.

Given a quintuple of real numbers $\left(c, h, c_{a}, h_{a}, c_{3}\right)$, there exists a unique irreducible representation of the Lie algebra $\mathscr{\mathscr { D }}$, denoted by $R\left(c, h, c_{a}, h_{a}, c_{3}\right)$ such that the central elements $c, c_{a}$, and $c_{3}$ operate as scalars (denoted by the same 
letters) and there exists a non-zero vector $v \in R\left(c, h, c_{a}, h_{a}, c_{3}\right)$ with the property

$$
\begin{gathered}
d_{n}(v)=0 \quad \text { and } \quad z^{n}(v)=0 \quad \text { for } n>0 \\
d_{0}(v)=h v, z^{0}(v)=h_{a} v
\end{gathered}
$$

A Verma module $\widetilde{R}\left(c, h, c_{a}, h_{a}, c_{3}\right)$ is defined in the usual way. It carries a unique Hermitian form $\langle\cdot \mid \cdot\rangle$ such that $d_{n}^{*}=d_{-n}, z^{n *}=z^{-n}$, and $\langle v \mid v\rangle=1$ for a highest weight vector $v$. This is called the contravariant Hermitian form. One has the induced contravariant Hermitian form on

$$
\tilde{R}\left(c, h, c_{a}, h_{a}, c_{3}\right) / \operatorname{Ker}\langle\cdot \mid \cdot\rangle=R\left(c, h, c_{a}, h_{a}, c_{3}\right) .
$$

(6.6) Theorem. The representation $R\left(c, h, c_{a}, h_{a}, c_{3}\right)$ is unitary (i.e. the contravariant Hermitian form is positive definite) in precisely the following cases:

I) $c_{a}=0$; then $c_{3}=0, h_{a}=0$, and $(c, h)$ is the highest weight of a unitary representation of $\mathrm{Vir}$ (the list is well-known).

II) $c_{a}>0$; then either

or

$$
c-\frac{12 c_{3}^{2}}{c_{a}} \geqq 2, \quad h-\frac{h_{a}^{2}+c_{3}^{2}}{2 c_{a}} \geqq 0,
$$

where $m \in \mathbb{Z}_{\geqq 0}$, and

$$
c-\frac{12 c_{3}^{2}}{c_{a}}=2-\frac{6}{(m+2)(m+3)},
$$

$$
h-\frac{h_{a}^{2}+c_{3}^{2}}{2 c_{a}}=\frac{[(m+3) r-(m+2) s]^{2}-1}{4(m+2)(m+3)}
$$

with $1 \leqq s \leqq r \leqq m+1, s, r \in \mathbb{Z}$.

One has the following eigenspace decomposition with respect to $d_{0}$ :

$$
\widetilde{R}\left(c, h, c_{a}, h_{a}, c_{3}\right)=\bigoplus_{n \in \mathbb{Z}_{\geqq 0}} \widetilde{R}_{h+n} ;
$$

since $d_{0}$ is selfadjoint, the eigenspaces $\widetilde{R}_{h+n}$ are orthogonal to each other. We denote by $\operatorname{det}_{n}\left(c, h, c_{a}, h_{a}, c_{3}\right)$ the determinant of the contravariant form restricted to $\widetilde{R}_{h+n}$ (it is defined up to a positive constant factor depending on the choice of the basis). Let $\sum_{n} p^{(2)}(n) q^{n}=\prod_{n \geqq 1}\left(1-q^{n}\right)^{-2}$.

(6.7) Theorem 2. Introduce the following polynomials in the 5 variables $c, h, c_{a}, h_{a}$, and $c_{3}$ :

and

$$
\begin{aligned}
\phi_{r, s}= & {\left[c_{a} h-\frac{1}{2} h_{a}^{2}-\frac{1}{2} c_{3}^{2}+\frac{1}{24}\left(r^{2}-1\right)\left(c c_{a}-14 c_{a}-12 c_{3}^{2}\right)+\frac{1}{2}(r s-1) c_{a}\right] } \\
& \cdot[r \leftrightarrow s]+\frac{1}{16}\left(r^{2}-s^{2}\right)^{2} c_{a}^{2} \quad \text { for } \quad r \neq s,
\end{aligned}
$$

$$
\phi_{r, r}=c_{a} h-\frac{1}{2} h_{a}^{2}-\frac{1}{2} c_{3}^{2}+\frac{1}{24}\left(r^{2}-1\right)\left(c c_{a}-2 c_{a}-12 c_{3}^{2}\right) .
$$

Then one has:

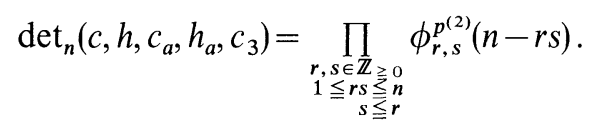


Proof. The proof of these two theorems is based on the following construction from string theory.

Let $L\left(c_{a}, h_{a}\right)$ be a highest weight representation of the oscillator algebra (6.2): $z^{m} \mapsto \alpha_{m}(m \in \mathbb{Z})$. Given $c_{3} \in \mathbb{R}$, this representation extends to the whole Lie algebra $\hat{\mathscr{D}}$, provided that $c_{a} \neq 0$, as follows:

This gives us

$$
\begin{gathered}
d_{n}=\frac{1}{c_{a}}\left(\frac{1}{2} \sum_{j \in \mathbb{Z}} \alpha_{-j} \alpha_{n+j}+\sqrt{-1} c_{3} n \alpha_{n}\right) \text { if } n \neq 0 \\
d_{0}=\frac{1}{c_{a}}\left(\frac{h_{a}^{2}+c_{3}^{2}}{2}+\sum_{j>0} \alpha_{-j} \alpha_{j}\right) .
\end{gathered}
$$

$$
L\left(c_{a}, h_{a}\right)=R\left(1+\frac{12 c_{3}^{2}}{c_{a}}, \frac{h_{a}^{2}+c_{3}^{2}}{2 c_{a}}, c_{a}, h_{a}, c_{3}\right) .
$$

Furthermore, a highest weight representation $V(c, h)$ of Vir extends trivially to $\widehat{\mathscr{D}}$, giving

$$
V(c, h)=R(c, h, 0,0,0) \text {. }
$$

From (6.8) and (6.9) we deduce, provided $c_{a} \neq 0$ :

$$
R\left(c, h, c_{a}, h_{a}, c_{3}\right)=L\left(c_{a}, h_{a}\right) \otimes V\left(c-1-\frac{12 c_{3}^{2}}{c_{a}}, h-\frac{h_{a}^{2}+c_{3}^{2}}{2 c_{a}}\right) .
$$

Theorem 1 in the case $c_{a} \neq 0$ follows from (6.10) and the known classification of unitary highest weight representations of Vir. [We use also the trivial fact that $L\left(c_{a}, h_{a}\right)$ is unitary iff $c_{a} \geqq 0$.]

Theorem 2 follows from the analogue of (6.10) for Verma modules and the known determinant formula for Vir. Now Theorem 1 in the case $c_{a}=0$ follows from Theorem 2 with $c_{a}=0$. Q.E.D.

(6.11) Remark. Theorems 1 and 2 in the case $c_{3}=0$ were obtained in [K], where one can also find all the related references.

Acknowledgements. We owe a debt to the ideas of [BMS, BM, M]. It is also a pleasure to thank R. Bott, I. M. Singer and especially D. Kazhdan for useful conversations. The second named author wishes to thank the Department of Mathematics of Brandeis and Harvard Universities for their support.

After this paper was completed we learned about [BS, Ko, KNTY] where some of our constructions are also discussed.

\section{References}

[AGR] Alvarez-Gaumé, L., Gomez, C., Reina, C.: Loop groups, Grassmannians and string theory. Phys. Lett. 190 B, 55-62 (1987)

[ACGH] Arbarello, E., Cornalba, M., Griffiths, P.A., Harris, J.: Geometry of algebraic curves, Vol. I. Berlin, Heidelberg, New York: Springer 1985, Vol. II (to appear)

[AC] Arbarello, E., Cornalba, M.: The Picard groups of the moduli spaces of curves. Topology 26 (2), 153-171 (1987)

[BM] Beilinson, A.A., Manin, Yu.I.: The Mumford form and the Polyakov measure in string theory. Commun. Math. Phys. 107, 359-376 (1986) 
[BMS] Beilinson, A.A., Manin, Yu.I., Schechtman, V.V.: Sheaves of the Virasoro and NeveuSchwarz algebras. Moscow University preprint, 1987

[BS] Beilinson, A.A., Schechtman, V.V.: Determinant bundles and Virasoro algebras (preprint)

[C] Colombeau, J.-F.: Differential calculus and holomorphy. Amsterdam: North-Holland 1982

[DJKM] Date, E., Jimbo, M., Kashiwara, M., Miwa, T.: Transformation groups for soliton equations. In: Nonlinear integrable systems classical theory and quantum theory. Singapore: World Scientific 1983, pp. 39-119

[H] Harer, J.: The second homology group of the mapping class group of an orientable surface. Invent. Math. 72, 221-239 (1983)

[KP] Kac, V.G., Peterson, D.H.: Spin and wedge representations of infinite dimensional Lie algebras and groups. Proc. Natl. Acad. Sci. USA 78, 3308-3312 (1981)

[K] Kac, V.G.: Highest weight representations of conformal current algebras. In: Geometrical methods in field theory. Singapore: World Scientific 1986, pp. 3-15

[KNTY] Kawamoto, N., Namikawa, Y., Tsuchiya, A., Yamada, Y.: Geometric realization of conformal field theory on Riemann surfaces. Commun. Math. Phys. (in press)

[KM] Knudsen, F., Mumford, D.: The projectivity of the moduli space of stable curves. I. Math. Scand. 39, 19-55 (1976)

[Ko] Kontzevich, M.L.: Virasoro algebra and Teichmüller spaces. Funct. Anal. Appl. 21, No. 2, 78-79 (1987)

[M] Manin, Yu.I.: Quantum string theory and algebraic curves. Berkeley I.C.M. talk, 1986

[Mu $\quad$ Mumford, D.: Stability of projective varieties. Enseign. Math. 23, 39-110 (1977)

[Mu 1] Mumford, D.: Towards an enumerative geometry of the moduli space of curves. In: Progress in Math., Vol. 36. Boston: Birkhäuser 1983

[PS] Pressley, A., Segal, G.: Loop groups. Oxford: Oxford University Press 1986

[R] Ruckle, W.H.: Sequence spaces. London: Pitnam 1981

[SW] Segal, G., Wilson, G.: Loop groups and equations of KdV type. Publ. Math. I.H.E.S. 61, 3-64 (1985)

Communicated by A. Jaffe

Received September 21, 1987; in revised form November 30, 1987 